Draft Version November 6, 2018

Typeset using IATEX twocolumn style in AASTeX61

\title{
THE UNIVERSALITY OF THE RAPID NEUTRON-CAPTURE PROCESS REVEALED BY A POSSIBLE DISRUPTED DWARF GALAXY STAR*
}

\author{
Andrew R. CASey ${ }^{1,2}$ and Kevin C. Schlaufman ${ }^{3}$
}

${ }^{1}$ School of Physics 83 Astronomy, Monash University, Clayton 3800, Victoria, Australia

${ }^{2}$ Faculty of Information Technology, Monash University, Clayton 3800, Victoria, Australia

${ }^{3}$ Department of Physics and Astronomy, Johns Hopkins University, 3400 N Charles St., Baltimore, MD 21218, USA

(Received 2017 September 1; Revised 2017 September 12; Accepted 2017 September 18)

\begin{abstract}
The rapid neutron-capture process or $r$-process is thought to produce the majority of the heavy elements $(Z>30)$ in extremely metal-poor stars. The same process is also responsible for a significant fraction of the heavy elements in the Sun. This universality of the $r$-process is one of its characteristic features as well as one of the most important clues to its astrophysical origin. We report the discovery of an extremely metal-poor field giant with $[\mathrm{Sr}, \mathrm{Ba} / \mathrm{H}] \approx-6.0$ and $[\mathrm{Sr}, \mathrm{Ba} / \mathrm{Fe}] \approx-3.0$, the lowest abundances of strontium and barium relative to iron ever observed. Despite its low abundances, the star 2MASS J151113.24-213003.0 has $[\mathrm{Sr} / \mathrm{Ba}]=-0.11 \pm 0.14$ and therefore its neutron-capture abundances are consistent with the main solar $r$-process pattern that has $[\mathrm{Sr} / \mathrm{Ba}]=-0.25$. It has been suggested that extremely low neutron-capture abundances are a characteristic of dwarf galaxies, and we find that this star is on a highly-eccentric orbit with apocenter $\gtrsim 100 \mathrm{kpc}$ that lies in the disk of satellites in the halo of the Milky Way. We show that other extremely metal-poor stars with low $[\mathrm{Sr}, \mathrm{Ba} / \mathrm{H}]$ and $[\mathrm{Sr}, \mathrm{Ba} / \mathrm{Fe}]$ plus solar $[\mathrm{Sr} / \mathrm{Ba}]$ tend to have orbits with large apocenters, consistent with a dwarf galaxy origin for this class of object. The nucleosynthesis event that produced the neutron-capture elements in 2MASS J151113.24-213003.0 must produce both strontium and barium together in the solar ratio. We exclude contributions from the $s$-process in intermediate-mass AGB or fast-rotating massive metal-poor stars, pair-instability supernovae, the weak $r$-process, and neutron-star mergers. We argue that the event was a Pop III or extreme Pop II core-collapse supernova explosion.
\end{abstract}

Keywords: Galaxy: halo — Galaxy: kinematics and dynamics — galaxies: dwarf — stars: abundances — stars: Population II — stars: Population III

Corresponding author: Andrew R. Casey

andrew.casey@monash.edu

* This paper includes data gathered with the 6.5 meter Magellan Telescopes located at Las Campanas Observatory, Chile. 


\section{INTRODUCTION}

The rapid neutron-capture or $r$-process occurs when nuclei experience a neutron flux so intense that the lag time between neutron-capture events is less than the corresponding $\alpha$ - or $\beta$-decay timescale (e.g., Burbidge et al. 1957; Cameron 1957a,b). Many astrophysical events have been identified as candidates to produce conditions favorable for the $r$-process, most (but by no means all) involving either core-collapse supernovae or neutron-star mergers. Chemical evolution models of the Milky Way suggest that common events that produce small amounts of $r$-process material and rare events that produce large amounts of $r$-process material are both possible (e.g., Qian 2000; Argast et al. 2004; Matteucci et al. 2014; Shen et al. 2015; van de Voort et al. 2015; Naiman et al. 2017).

While the astrophysical site of the $r$-process is still hotly debated, its observational signature in the abundances of the neutron-capture elements (i.e., $Z>30$ ) has been seen from the Sun all the way to the most ancient stars known. Whereas the abundances of the neutron-capture elements seems to be decoupled from the abundances of the light (i.e., $Z \leq 13$ ), $\alpha$, and iron-peak (i.e., $21 \leq Z \leq 30$ ) elements in extremely metal-poor stars, no star completely lacking in neutroncapture elements has yet been found (e.g., Gilroy et al. 1988; McWilliam et al. 1995a,b; Norris et al. 1996; Ryan et al. 1996; Cayrel et al. 2004; François et al. 2007; Cohen et al. 2013; Yong et al. 2013; Roederer 2013; Roederer et al. 2014).

Even with the highest-quality data practically attainable, only the abundances of a few neutron-capture elements can be measured from high-resolution spectra of ordinary extremely metal-poor stars. The most frequently measurable neutron-capture elements with the strongest atomic transitions in the visible - and the only ones observable in neutron-capture poor stars - are strontium and barium. In extremely metal-poor stars, strontium $(Z=38$ and $A=86-88)$ is representative of the first $r$-process peak while barium $(Z=56$ and $A=134-138)$ is representative of the second peak. Though both elements can be produced by the slow neutron-capture or $s$-process in asymptotic giant branch (AGB) stars, observations of metal-poor stars do not show contributions from the $s$-process for stars with $[\mathrm{Fe} / \mathrm{H}] \lesssim-2.8$ (Simmerer et al. 2004).

The relative abundances of the neutron-capture elements in the first and second $r$-process peaks can be used to diagnose the properties and astrophysical site of the $r$-process that produced the heavy elements in metalpoor stars. For most metal-poor stars, [Sr/Ba] is inversely correlated with $[\mathrm{Ba} / \mathrm{Fe}]$. The neutron-capture el- ements in stars with $[\mathrm{Sr} / \mathrm{Ba}] \sim 1$ have been suggested to have been formed by the "weak" $r$-process. In this scenario, the neutron flux is too weak to create the heaviest neutron-capture elements such that the first $r$-process peak is produced but the second peak is not (e.g., Truran et al. 2002). On the other hand, the neutron-capture elements in stars with $[\mathrm{Sr} / \mathrm{Ba}] \sim-1$ were produced by an intense neutron flux that caused the $r$-process to seemingly skip the first peak and strongly populate the second. Metal-poor stars with $[\mathrm{Sr} / \mathrm{Ba}] \sim 0$ are consistent with the "main" $r$-process responsible for a significant fraction of the neutron-capture elements in the Sun. Interestingly, there is a hint that in the most neutron-capture poor stars the inverse correlation between $[\mathrm{Ba} / \mathrm{Fe}]$ and $[\mathrm{Sr} / \mathrm{Ba}]$ is broken and $[\mathrm{Sr} / \mathrm{Ba}] \sim 0$ is consistent with the main $r$-process (e.g., François et al. 2007; Lai et al. 2008). In other words, the same main $r$-process seems to be responsible both for the Sun and for the stars closest in time to the first $r$-process events.

It is easier to model the chemical evolution of classical and ultra-faint dwarf (UFD) galaxies than the Milky Way, so neutron-capture abundances in dwarf galaxies are especially useful in the effort to identify the astrophysical site of the $r$-process. Until recently, most observational data suggested that at constant metallicity the metal-poor stars in UFD galaxies had neutroncapture abundances below those of stars in the halo of the Milky Way. To date, neutron-capture poor giants (i.e., $[\mathrm{Sr}, \mathrm{Ba} / \mathrm{Fe}]<-1$ ) have been observed in the UFD galaxies Boötes II (Ji et al. 2016d), Coma Berenices (Frebel et al. 2010), Leo IV (Simon et al. 2010; François et al. 2016), Reticulum II (Roederer et al. 2016), Segue 1 (Frebel et al. 2014), Segue 2 (Roederer \& Kirby 2014), Triangulum II (Kirby et al. 2017), Tucana II (Ji et al. 2016b), and Ursa Major II (Frebel et al. 2010). Such stars also occasionally occur in classical dwarfs like Carina (Venn et al. 2012), Draco (Fulbright et al. 2004; Cohen \& Huang 2009), Hercules (Koch et al. 2014), and Sculptor (Starkenburg et al. 2013; Jablonka et al. 2015; Simon et al. 2015a). These observations lead to the suggestion that low neutron-capture abundances are a signature of dwarf galaxy chemical evolution (e.g., Frebel \& Norris 2015). This idea is consistent with the hypothesis that UFD galaxies are the closest known objects to the first galaxies, possibly hosting stars without any neutron-capture elements at all.

The recent discovery of the UFD galaxy Reticulum II has provided another important clue to the origin of the $r$-process (Koposov et al. 2015a,b; Bechtol et al. 2015; Simon et al. 2015b; Walker et al. 2015). Most stars in Reticulum II are extremely enriched in neutroncapture elements (Ji et al. 2016a,c; Roederer et al. 
2016). The extreme enrichment disfavors $r$-process nucleosynthesis in small amounts in individual supernovae and instead favors the injection of a large amount of neutron-capture elements in a rare event like a magnetorotationally driven supernova, neutron star-neutron star merger, or black hole-neutron star merger. Given the relative isolation and chemically-primitive nature of Reticulum II, it provides the first unambiguous evidence for $r$-process nucleosynthesis in a rare event that produced a large amount of neutron-capture elements.

All currently known dwarf galaxies are more than 20 kpc from the Sun though, so even metal-poor giants are usually too faint to collect high-resolution spectra with a sufficient signal-to-noise ratio $(\mathrm{S} / \mathrm{N})$ to measure the abundances of strontium and barium in neutron-capture poor stars. Much more often it is only possible to set upper limits. While upper limits on neutron-capture abundances for dwarf galaxy stars are consistent with the lowest neutron-capture abundances seen in the field (e.g., Roederer 2013), in dwarf galaxies it is impossible to differentiate stars with no neutron-capture elements from stars with little neutron-capture elements. That test could only be done with brighter, nearby extremely metal-poor giants that are also neutron-capture poor.

Here we report the discovery of 2MASS J151113.24213003.0, an extremely metal-poor star with the lowest abundance of neutron-capture elements relative to iron ever observed. We describe our observations in Section 2 , and the analysis of those data in Section 3. In Section 4 we discuss the origin of 2MASS J151113.24-213003.0 and the mechanisms that could have produced the chemical abundance pattern we observe. We conclude in Section 5 .

\section{OBSERVATIONS}

We identified 2MASS J151113.24-213003.0 as a candidate metal-poor star because it satisfies criteria (1)(4) of the photometric selection described in Schlaufman \& Casey (2014). ${ }^{1}$ We initially observed the star on 22 June 2014 - the first night of Magellan observations for the pilot "Best and Brightest" programusing the Magellan Inamori Kyocera Echelle (MIKE) spectrograph on the Magellan Clay Telescope at Las Campanas Observatory (Bernstein et al. 2003; Shectman \& Johns 2003). Our moderate signal-to-noise ratio $(\mathrm{S} / \mathrm{N} \approx 30)$ high-resolution $(\mathcal{R} \approx 28,000)$ snapshot spectrum confirmed that 2MASS J151113.24-213003.0 is an extremely metal-poor giant. A preliminary detailed chemical abundance analysis revealed remarkably

\footnotetext{
1 We note that 2MASS J151113.24-213003.0 also satisfies the full selection as defined in Schlaufman \& Casey (2014).
}

low levels of strontium and barium plus no detectable quantities of other neutron-capture elements.

This peculiar chemical pattern prompted us to acquire a high-quality follow-up spectrum. We therefore observed 2MASS J151113.24-213003.0 with MIKE a second time during evening twilight of 1 August 2015. The analysis presented here is based on those observations. We used the $0.35^{\prime \prime}$ slit, which provides a spectral resolution of $\mathcal{R} \approx 83,000$ in the blue and $\mathcal{R} \approx 65,000$ in the red. We obtained three exposures of 1,200 seconds each at airmass $<1.03$ in clear conditions with variable seeing between $0.5^{\prime \prime}$ and $1.7^{\prime \prime}$. Calibration frames were taken in the afternoon (e.g., biases, quartz and "milky" flat fields, ThAr lamp frames). The signal-to-noise ratio of the stacked spectrum reaches $\mathrm{S} / \mathrm{N} \approx 130 \mathrm{pixel}^{-1}$ at $345 \mathrm{~nm}$ on the blue side, and $\mathrm{S} / \mathrm{N} \approx 280$ pixel $^{-1}$ at 500 $\mathrm{nm}$ on the red side.

\section{ANALYSIS}

We reduced the raw spectra and calibration frames using the CarPy software package (Kelson 2003; Kelson et al. 2014). The first step in our analysis of the extracted $1 \mathrm{D}$ spectrum was to place it in the rest frame. We used a spline function to continuum normalize the echelle order that includes the Ca II near-infrared triplet. We then measured the radial velocity by cross correlating the normalized order with a rest-frame normalized spectrum of the metal-poor giant star HD 122563. Using this line-of-sight velocity, we shifted all echelle orders to the rest frame without resampling. After applying the heliocentric correction ${ }^{2}$, we find a radial velocity of $v_{\mathrm{rad}}=14.4 \pm 1.0 \mathrm{~km} \mathrm{~s}^{-1}$.

\subsection{Stellar Parameters}

It is well established that the spectroscopic stellar parameters of metal-poor giant stars suffer from systematic uncertainties due in part to the violation of the assumptions of local thermodynamic equilibrium (LTE; e.g., Korn et al. 2003). These effects are particularly noticeable in the classical excitation/ionization balance approach, which results in significant biases in effective temperature $T_{\text {eff }}$, surface gravity $\log g$, and metallicity $[\mathrm{Fe} / \mathrm{H}]$ (e.g., Frebel et al. 2013a). For this reason, we performed a complementary determination of the spectroscopic stellar parameters of 2MASS J151113.24-

\footnotetext{
2 In Schlaufman \& Casey (2014) we incorrectly reported the heliocentric radial velocity of 2MASS J151113.24-213003.0 as $31.0 \mathrm{~km} \mathrm{~s}^{-1}$. During the course of this work we discovered that the radial velocity measurements reported in Schlaufman \& Casey (2014) did not include a correction for heliocentric motion. An erratum is in preparation. The heliocentric radial velocity measured on 2014 June 22 is $13.4 \pm 1.0 \mathrm{~km} \mathrm{~s}^{-1}$.
} 
213003.0 to minimize the effect of systematic uncertainty.

The complementary approach makes use of the wings of Balmer lines present in our spectrum, as Balmer line wings are extremely sensitive to $T_{\text {eff }}$ given a high-S/N, high-resolution spectrum. Because they have little degeneracy with other astrophysical parameters, the wings of Balmer lines are arguably the most accurate way of spectroscopically determining $T_{\text {eff }}$ when a high-quality spectrum is available (e.g., Fuhrmann 1998; Barklem et al. 2003). A limitation is that the profile wings are strongly degenerate with the continuum, and to a lesser extent, the residual line-of-sight velocity and the instrument resolution (Korn 2002).

In order to account for the correlations between astrophysical and nuisance parameters, we constructed a generative model for the data. We used the grids of continuum-normalized hydrogen line profiles computed by Barklem et al. (2003). ${ }^{3}$ However, our tests revealed that interpolating fluxes between grid points-even with high-order polynomial or spline interpolationproduced systematic offsets in flux at the order of $1.0 \%$. The bias direction and magnitude varied depending on position in the Hertzprung-Russell diagram. While this is a small offset, the sensitivity of the Balmer line wings implies that this could propagate to a bias in $T_{\text {eff }}$ of about $50 \mathrm{~K}$. Moreover, the number of pixels used when comparing the model and the data here is relatively small, implying that single-pixel systematics introduced by interpolation could have a noticeable effect on the inferred stellar parameters.

Consequently, we chose not to interpolate the grid of pre-computed Balmer-line profiles. Instead we calculated the likelihood at each grid point and marginalized over the nuisance parameters described above. Specifically, for each pre-computed Balmer line profile we modeled the continuum by multiplying the continuumnormalized flux with a third-order polynomial with coefficients $\left\{c_{m}\right\}_{m=1}^{M}$, and we included a term for the residual radial velocity $v_{\text {res }}$.

We constructed a mask to exclude the core of each line, as well as any stellar or telluric absorption in the neighboring wings. For each Balmer line model we optimized the nuisance parameters by minimizing the negative loglikelihood $-\log \mathcal{L}$. We then used the Gaussian approximation of a $K$-dimensional integral $(2 \pi)^{K / 2} / \sqrt{\operatorname{det} \boldsymbol{\Sigma}}$ (based on the formal covariance matrix $\boldsymbol{\Sigma}$ ) to marginalize over the nuisance parameters at every grid point.

\footnotetext{
3 http://www.astro.uu.se/ barklem/
}

We tested this approach by fitting the $\mathrm{H}-\alpha, \mathrm{H}-\beta, \mathrm{H}-$ $\gamma$, and $\mathrm{H}-\delta$ lines separately using unnormalized echelle orders of the well-studied metal-poor stars HD 122563 and HD 140283. For these stars, we found the H- $\beta$ line to be the best indicator of $T_{\text {eff }}{ }^{4}$ For HD 122563, our marginalized 1D posterior peaks at $T_{\text {eff }}=4543_{-130}^{+90} \mathrm{~K}$. For HD 140283, the posterior peaks at $T_{\text {eff }}=5448_{-70}^{+80} \mathrm{~K}$. These results are in excellent agreement with bolometric temperatures determined using interferometric radii (Heiter et al. 2015). That approach suggests that HD 122563 has $T_{\text {eff }}=4587 \pm 60 \mathrm{~K}$, a central value just 44 $\mathrm{K}$ hotter than our measurement. A similar calculation indicates that HD 140283 has $T_{\text {eff }}=5522 \pm 105 \mathrm{~K}, 74 \mathrm{~K}$ warmer than our central value. In both cases, the temperatures we find for HD 122563 and HD 140283 are within one standard deviation of either the bolometric or the spectroscopic uncertainty. Confident in our approach, we report the effective temperature of 2MASS J151113.24-213003.0 as $T_{\text {eff }}=4602_{-156}^{+199} \mathrm{~K}$ (Figure 1). We note that the posteriors on $\log g$ and $[\mathrm{M} / \mathrm{H}]$ from fitting the $\mathrm{H}-\beta$ line were uninformative: they suggested 2MASS J151113.24-213003.0 is more likely a metal-poor giant than a metal-rich dwarf, but the probability distribution function is flat.

We determined $\log g,[\mathrm{Fe} / \mathrm{H}]$, and microturbulence $\xi$ by measuring the strengths of Fe I and Fe II atomic absorption lines. We normalized individual rest-frame echelle orders by fitting a spline function to continuum regions, then resampled and stacked individual orders onto a common dispersion array to produce a contiguous rest-frame normalized spectrum from $331 \mathrm{~nm}$ to $916 \mathrm{~nm}$. Following the algorithm described in Casey (2014), we measured the strengths of individual absorption lines by fitting Gaussian profiles. All profiles were scrutinized to identify and discard obviously spurious measurements. We take the strengths and associated atomic data for all transitions from Roederer et al. (2010). We report both our measurements and the atomic data we used in Table 3.

Given our $T_{\text {eff }}$ inferred from the wings of the $\mathrm{H}-\beta$ line, we performed an ionization balance to determine $\log g$, $[\mathrm{Fe} / \mathrm{H}]$, and $\xi$. We used the $\alpha$-enhanced model atmospheres from Castelli \& Kurucz (2004) and the 2011 version of MOOG (Sneden 1973; Sobeck et al. 2011) to calculate line abundances. With $T_{\text {eff }}=4602 \mathrm{~K}$ fixed, we find $\log g=0.84,[\mathrm{Fe} / \mathrm{H}]=-3.05$, and $\xi=2.22 \mathrm{~km} \mathrm{~s}^{-1}$. However, because the surface gravities of metal-poor giant stars are routinely underestimated due to non-LTE effects, we chose to adopt $\log g=1.02$ from a 10 Gyr $\alpha$ -

\footnotetext{
${ }^{4}$ A similar process leading to the same conclusion can be found in Barklem et al. (2002).
} 


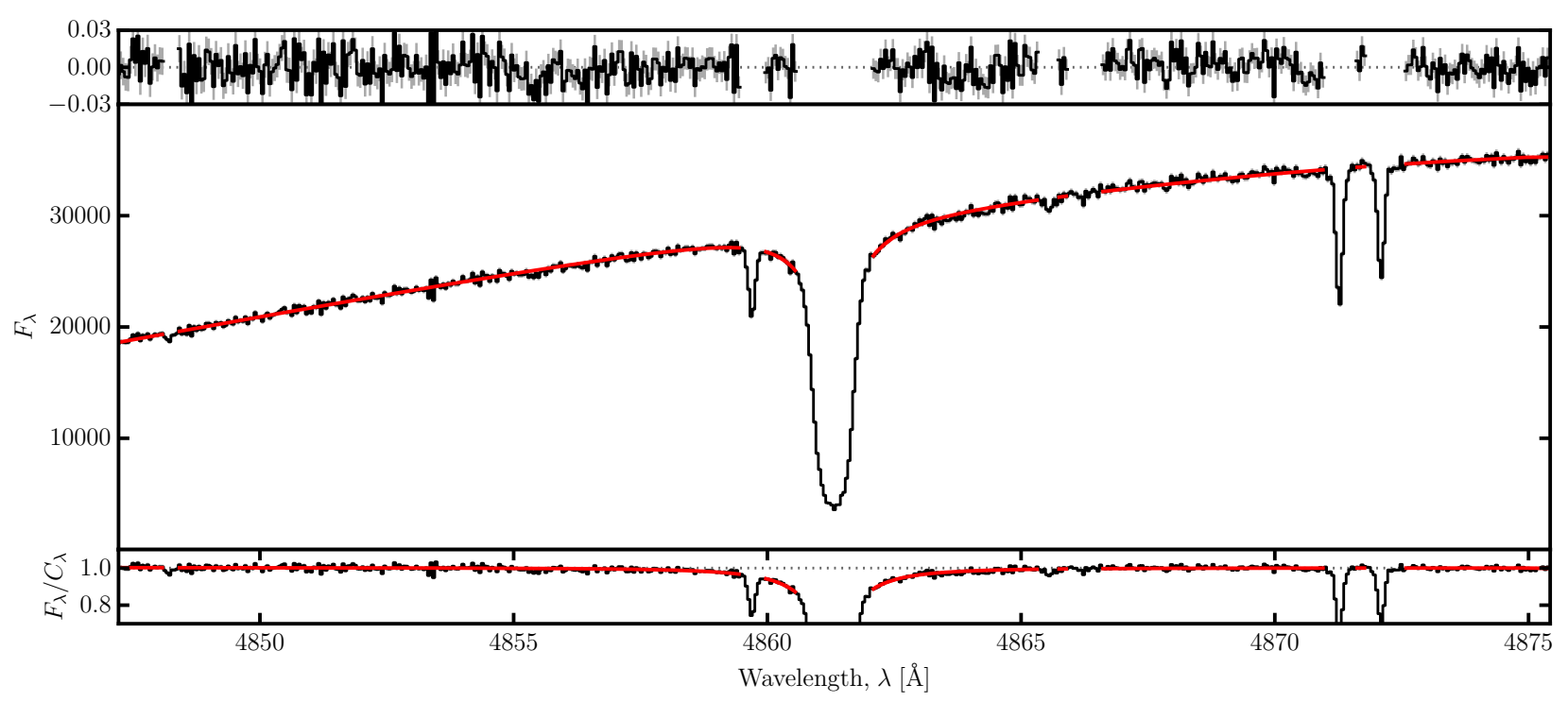

Figure 1. Magellan/MIKE spectrum of 2MASS J151113.24-213003.0 surrounding the H- $\beta$ line. The center panel is the spectrum itself (where the flux have arbitrary units), the bottom panel is the spectrum divided by the inferred continuum, and the top panel is the residual difference between the data and the model. We also plot in red the optimized model closest grid point to the maximum a posteriori solution.

enhanced Dartmouth isochrone of $[\mathrm{Fe} / \mathrm{H}]=-2.5$ (Dotter et al. 2008). This produced just a 0.02 dex decrease in the mean metallicity to $[\mathrm{Fe} / \mathrm{H}]=-3.07$ and a $0.05 \mathrm{~km}$ $\mathrm{s}^{-1}$ decrease in microturbulence to $\xi=2.17 \mathrm{~km} \mathrm{~s}^{-1}$. It also caused $(\langle\mathrm{Fe} \mathrm{I}\rangle-\langle\mathrm{Fe}$ II $\rangle)$ to decrease to -0.07 dex. We report the adopted parameters and associated uncertainties in Table 1 . These values are in good agreement with the stellar parameters derived from our reconnaissance spectrum and reported in Schlaufman \& Casey (2014). The effective temperature inferred from the $\mathrm{H}-\beta$ line is $115 \mathrm{~K}$ cooler than the Schlaufman \& Casey (2014) measurement determined solely by excitation balance, and the 0.1 dex changes in $\log g$ and $[\mathrm{Fe} / \mathrm{H}]$ are consistent with the change in inferred temperature. These differences are within the quoted 1- $\sigma$ uncertainties of both Schlaufman \& Casey (2014) and this study.

\subsection{Detailed Chemical Abundances}

We report the detailed chemical abundances of 2MASS J151113.24-213003.0 in Table 2. For most elements, we determined individual chemical abundances from measured equivalent widths of unblended atomic lines. However, we adopted a synthesis approach for molecular features (e.g., $\mathrm{CH}$ ) and for atomic transitions with strong isotopic splitting or hyperfine structure (specifically scandium, manganese, cobalt, and copper). We used molecular data for $\mathrm{CH}$ from Masseron et al. (2014) as well as hyperfine/isotopic splitting data from Kurucz \& Bell (1995) for the iron-peak elements, Biémont et al. (1999) for barium, and Lawler et al. (2001a,b) for europium and lanthanum. We assume $r$ process only isotopic fractions from Sneden et al. (2008).

For some elements we were only able to measure the abundance from a single atomic transition. One example is K I, where our abundance is calculated solely from the $767 \mathrm{~nm}$ line because the $770 \mathrm{~nm}$ line was contaminated by strong telluric absorption. Similarly, only upper limits were possible for many of the elemental abundances. This includes lithium, oxygen, cobalt, copper, and all of the neutron-capture elements except strontium and barium. Upper limits were calculated from the strongest absorption line available for each species. Most of our derived abundance limits are not informative despite the high quality of our spectrum.

\subsection{Dynamics}

We combined the stellar parameters inferred in Section 3.1 with isochrones and multi-band photometry to compute a posterior distance distribution to 2MASS J151113.24-213003.0. We used $B$ and $V$ photometry from APASS DR9 (Henden et al. 2015); $J, H$, and $K_{s}$ photometry from 2MASS (Skrutskie et al. 2006); and $W_{1}, W_{2}$, and $W_{3}$ photometry from WISE (Wright et al. 2010). Apparent magnitudes were de-reddened using the Schlegel et al. (1998) dust maps as updated by Schlafly \& Finkbeiner (2011). We used the MIST isochrone grid (Dotter 2016; Choi et al. 2016; Paxton et al. 2011, 2013, 2015) supplied with the Python isochrones package (Morton 2015). This leads us to an inferred distance of $d_{\odot}=7.5_{-0.5}^{+0.6} \mathrm{kpc}$. 
Table 1. Parameters of 2MASS J151113.24-213003.0

\begin{tabular}{|c|c|}
\hline Property & Value \\
\hline Right ascension $\alpha$ (J2000) & 151113.24 \\
\hline Declination $\delta(\mathrm{J} 2000)$ & -213003.0 \\
\hline$V$-band apparent magnitude ${ }^{\mathrm{a}}$ & $12.66 \pm 0.06$ \\
\hline$J-K_{s}$ color $^{\mathrm{b}}$ & 0.62 \\
\hline Reddening $^{\mathrm{c}} E(B-V)$ & 0.099 \\
\hline Effective temperature $T_{\text {eff }}$ & $4602_{-156}^{+199} \mathrm{~K}$ \\
\hline Surface gravity $\log g$ & $1.02_{-0.15}^{+0.15}$ \\
\hline Metallicity $[\mathrm{Fe} / \mathrm{H}]$ & $-3.07 \pm 0.10$ \\
\hline Microturbulence $\xi$ & $2.17 \pm 0.10 \mathrm{~km} \mathrm{~s}^{-1}$ \\
\hline Radial velocity $v_{\text {rad }}$ & $14.4 \pm 1.0 \mathrm{~km} \mathrm{~s}^{-1}$ \\
\hline Proper motion $^{\mathrm{d}}$ in $\alpha\left(\mu_{\alpha} \cos \delta\right)$ & 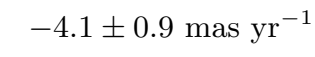 \\
\hline Proper motion ${ }^{\mathrm{d}}$ in $\delta\left(\mu_{\delta}\right)$ & 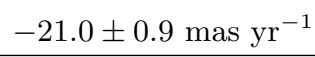 \\
\hline
\end{tabular}

\section{Informed analysis (preferred)}

Heliocentric distance $d_{\odot}$

$$
7.1_{-0.5}^{+0.6} \mathrm{kpc}
$$

Galactocentric distance $d_{\mathrm{GC}}$

$$
4.7_{-0.1}^{+0.1} \mathrm{kpc}
$$

Max height above Galactic plane $z_{\max }$

$$
106.6_{-29.1}^{+22.8} \mathrm{kpc}
$$

Apocenter $r_{\text {apo }}$

$$
125.2_{-35.5}^{+31.7} \mathrm{kpc}
$$

$$
4.3_{-0.1}^{+0.2} \mathrm{kpc}
$$

Eccentricity $e$

$0.93_{-0.02}^{+0.01}$

\section{Uninformed analysis}

Heliocentric distance $d \odot$

Galactocentric distance $d_{\mathrm{GC}}$

$4.2_{-1.0}^{+1.0} \mathrm{kpc}$

Max height above Galactic plane $z z_{\max }$

$5.2_{-0.4}^{+0.5} \mathrm{kpc}$

$7.7_{-1.8}^{7.9} \mathrm{kpc}$

Apocenter $r_{\text {apo }}$

$9.7_{-2.8}^{+12.3} \mathrm{kpc}$

Pericenter $r_{\text {peri }}$

$4.0_{-0.2}^{+0.3} \mathrm{kpc}$

Eccentricity $e$

$0.53_{-0.14}^{+0.24}$

${ }^{a}$ Photometry from APASS DR9 (Henden et al. 2015)

${ }^{b}$ Photometry from 2MASS (Skrutskie et al. 2006)

${ }^{c}$ Using the Schlegel et al. (1998) dust maps as updated by Schlafly \& Finkbeiner (2011)

$d_{\text {Proper motions from UCAC5 (Zacharias et al. 2017) }}$

After considering the available proper motions from HSOY (Altmann et al. 2017), PPMXL (Roeser et al. 2010), SPM4 (Girard et al. 2011), UCAC4 (Zacharias et al. 2013), and UCAC5 (Zacharias et al. 2017), we adopted the proper motions from UCAC5. UCAC5 in-

\begin{tabular}{|c|c|c|c|c|c|}
\hline Species & $\mathrm{N}$ & $\log _{\epsilon}(\mathrm{X})$ & $\sigma^{1}$ & {$[\mathrm{X} / \mathrm{H}]$} & {$[\mathrm{X} / \mathrm{Fe} \mathrm{I}]$} \\
\hline Li I & 1 & $<-0.25$ & $\ldots$ & $<-1.30$ & $<1.78$ \\
\hline $\mathrm{C}(\mathrm{CH})$ & & 4.80 & 0.10 & -3.63 & -0.55 \\
\hline O I & 1 & $<6.92$ & $\ldots$ & $<-1.77$ & $<1.31$ \\
\hline $\mathrm{Na} I$ & 1 & 3.63 & $\ldots$ & -2.61 & 0.47 \\
\hline Mg I & 11 & 5.08 & 0.09 & -2.52 & 0.55 \\
\hline $\mathrm{Al}$ I & 1 & 2.79 & $\ldots$ & -3.67 & -0.59 \\
\hline Si I & 2 & 5.09 & 0.13 & -2.42 & 0.66 \\
\hline $\mathrm{K}$ I & 1 & 2.19 & $\cdots$ & -2.84 & 0.24 \\
\hline $\mathrm{Ca} \mathrm{I}$ & 17 & 3.36 & 0.06 & -2.98 & 0.10 \\
\hline Sc I & 6 & -0.05 & 0.07 & -3.20 & -0.13 \\
\hline Sc II & 7 & -0.01 & 0.12 & -3.16 & -0.09 \\
\hline Ti II & 36 & 1.77 & 0.13 & -3.18 & -0.11 \\
\hline V II & 1 & 1.10 & $\cdots$ & -2.83 & 0.25 \\
\hline Cr I & 13 & 2.16 & 0.17 & -3.48 & -0.40 \\
\hline Cr II & 2 & 2.63 & 0.02 & -3.01 & 0.06 \\
\hline Mn I & 7 & 1.74 & 0.09 & -3.69 & -0.62 \\
\hline Mn II & 1 & 1.76 & $\ldots$ & -3.67 & -0.59 \\
\hline $\mathrm{Fe} \mathrm{I}$ & 223 & 4.42 & 0.12 & -3.08 & 0.00 \\
\hline $\mathrm{Fe}$ II & 23 & 4.49 & 0.12 & -3.01 & 0.07 \\
\hline Co I & 1 & $<2.91$ & $\cdots$ & $<-2.08$ & $<1.00$ \\
\hline Ni I & 9 & 3.12 & 0.12 & -3.10 & -0.02 \\
\hline $\mathrm{Cu} \mathrm{I}$ & 1 & $<1.36$ & $\cdots$ & $<-2.83$ & $<0.25$ \\
\hline Zn I & 2 & 1.51 & 0.01 & -3.05 & 0.03 \\
\hline $\mathrm{Ga} I$ & 1 & $<0.74$ & $\cdots$ & $<-2.30$ & $<0.77$ \\
\hline $\mathrm{Rb}$ I & 1 & $<1.26$ & $\ldots$ & $<-1.26$ & $<1.82$ \\
\hline Sr II & 2 & -3.04 & 0.17 & -5.91 & -2.84 \\
\hline Y II & 1 & $<-1.62$ & $\ldots$ & $<-3.83$ & $<-0.76$ \\
\hline Zr II & 1 & $<-1.29$ & $\cdots$ & $<-3.87$ & $<-0.80$ \\
\hline $\mathrm{Nb}$ II & 1 & $<-0.37$ & $\ldots$ & $<-1.83$ & $<1.25$ \\
\hline Mo I & 1 & $<-0.70$ & $\cdots$ & $<-2.58$ & $<0.50$ \\
\hline Sn I & 1 & $<0.71$ & $\ldots$ & $<-1.33$ & $<1.75$ \\
\hline Ba II & 1 & -3.62 & $\cdots$ & -5.80 & -2.72 \\
\hline La II & 1 & $<-2.68$ & $\ldots$ & $<-3.78$ & $<-0.70$ \\
\hline Ce II & 1 & $<-1.25$ & $\cdots$ & $<-2.83$ & $<0.25$ \\
\hline Pr II & 1 & $<-1.36$ & $\ldots$ & $<-2.08$ & $<1.00$ \\
\hline $\mathrm{Nd}$ II & 1 & $<-1.91$ & $\ldots$ & $<-3.33$ & $<-0.25$ \\
\hline Sm II & 1 & $<-1.62$ & $\ldots$ & $<-2.58$ & $<0.50$ \\
\hline Eu II & 1 & $<-2.98$ & $\ldots$ & $<-3.50$ & $<-0.42$ \\
\hline Gd II & 1 & $<-2.01$ & $\cdots$ & $<-3.08$ & $<0.00$ \\
\hline $\mathrm{Tb}$ II & 1 & $<-2.28$ & $\cdots$ & $<-2.58$ & $<0.50$ \\
\hline Dy II & 1 & $<-1.98$ & $\cdots$ & $<-3.08$ & $<0.00$ \\
\hline Tm II & 1 & $<-1.98$ & $\cdots$ & $<-2.08$ & $<1.00$ \\
\hline Yb II & 1 & $<-3.24$ & $\ldots$ & $<-4.08$ & $<-1.00$ \\
\hline Hf II & 1 & $<-1.48$ & $\cdots$ & $<-2.33$ & $<0.75$ \\
\hline Ir I & 1 & $<-0.45$ & $\cdots$ & $<-1.83$ & $<1.25$ \\
\hline $\mathrm{Pb} \mathrm{I}$ & 1 & $<-0.83$ & $\ldots$ & $<-2.58$ & $<0.50$ \\
\hline
\end{tabular}
corporates Gaia DR1 astrometry to improve the UCAC4 proper motions and currently provides the best avail-
Table 2. Chemical abundances of 2MASS J151113.24-213003.0

1 This is the statistical uncertainty (standard deviation) of the line abundance for each species. We estimate the systematic uncertainty at 0.10 dex for all elements, which we recommend be added in quadrature. 
able proper motions for bright stars not included in the Tycho-Gaia Astrometric Solution (Michalik et al. 2015; Lindegren et al. 2016). As a result, UCAC5 has the most precise proper motions and reports $\mu_{\alpha \cos \delta}=-4.1 \pm 0.9$

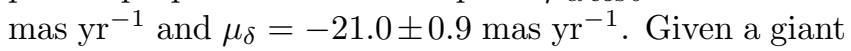
star with $d_{\odot} \approx 7 \mathrm{kpc}$, a proper motion in excess of 20 mas $\mathrm{yr}^{-1}$ implies a significant transverse velocity. We note that all of the proper motions catalogs we checked report similarly high proper motions in declination. ${ }^{5}$ In fact, the UCAC5 proper motions we adopt are slightly smaller in magnitude than the values reported in other catalogs.

We sample 1,000 Monte Carlo realizations from the $d_{\odot}$ posterior and the uncertainty distributions of $v_{\mathrm{rad}}$, $\mu_{\alpha \cos \delta}$, and $\mu_{\delta}$ under the assumption that they are normally distributed. We used each Monte Carlo realization as initial conditions for an orbit and integrated it forward 10 Gyr in a Milky Way-like potential using galpy (Bovy 2015). We adopted the MWPotential2014 described by Bovy (2015). In that model, the bulge is parameterized as a power-law density profile that is exponentially cut-off at $1.9 \mathrm{kpc}$ with a power-law exponent of -1.8 . The disk is represented by a Miyamoto-Nagai potential with a radial scale length of $3 \mathrm{kpc}$ and a vertical scale height of 280 pc (Miyamoto \& Nagai 1975). The halo is modeled as a Navarro-Frenk-White halo with a scale length of $16 \mathrm{kpc}$ (Navarro et al. 1996). We set the solar distance to the Galactic center as $R_{0}=8 \mathrm{kpc}$ and the circular velocity at the Sun to $V_{0}=220 \mathrm{~km} \mathrm{~s}^{-1}$ (Bovy et al. 2012). We find that 2MASS J151113.24213003.0 is approaching pericenter $\left(r_{\text {peri }}=4.3_{-0.1}^{+0.2} \mathrm{kpc}\right)$ on a highly-eccentric orbit $\left(e=0.93_{-0.02}^{+0.01}\right)$. We plot projections of galactic position $(x, y, z)$ from all realizations in Figure 2.

We repeated these inferences using a less-constrained distance prior to gauge the impact of our inferred stellar parameters. That is, we repeated the analysis described above with all available multi-band photometry, but with no constraint on reddening and with increased uncertainties on our spectroscopic stellar parameters. We imagined that all we knew about 2MASS J151113.24213003.0 was that it was an extremely metal-poor giant and set $T_{\text {eff }}=4750 \pm 250 \mathrm{~K}, \log g=1.5 \pm 0.5$, and

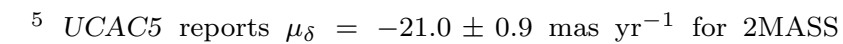
J151113.24-213003.0. That value is consistent with the HSOY $\left(-22.2 \pm 2.1\right.$ mas yr $\left.^{-1}\right)$, PPMXL $\left(-24.3 \pm 3.9{\left.\text { mas } \mathrm{yr}^{-1}\right), \text { SPM4 }}^{-1}\right.$ $\left(-22.2 \pm 1.3\right.$ mas $\left.\mathrm{yr}^{-1}\right)$, and UCAC4 $\left(-21.7 \pm 1.4 \mathrm{mas} \mathrm{yr}^{-1}\right)$ values. Indeed, all five catalogs agree within the 1- $\sigma$ uncertainty quoted by each catalog, and all catalogs consistently report $\mu_{\delta}$ at a level exceeding $6 \sigma$. Because 2MASS J151113.24-213003.0 is bright $(V=12.66)$, none of these entries are likely to be in error due to misidentification.
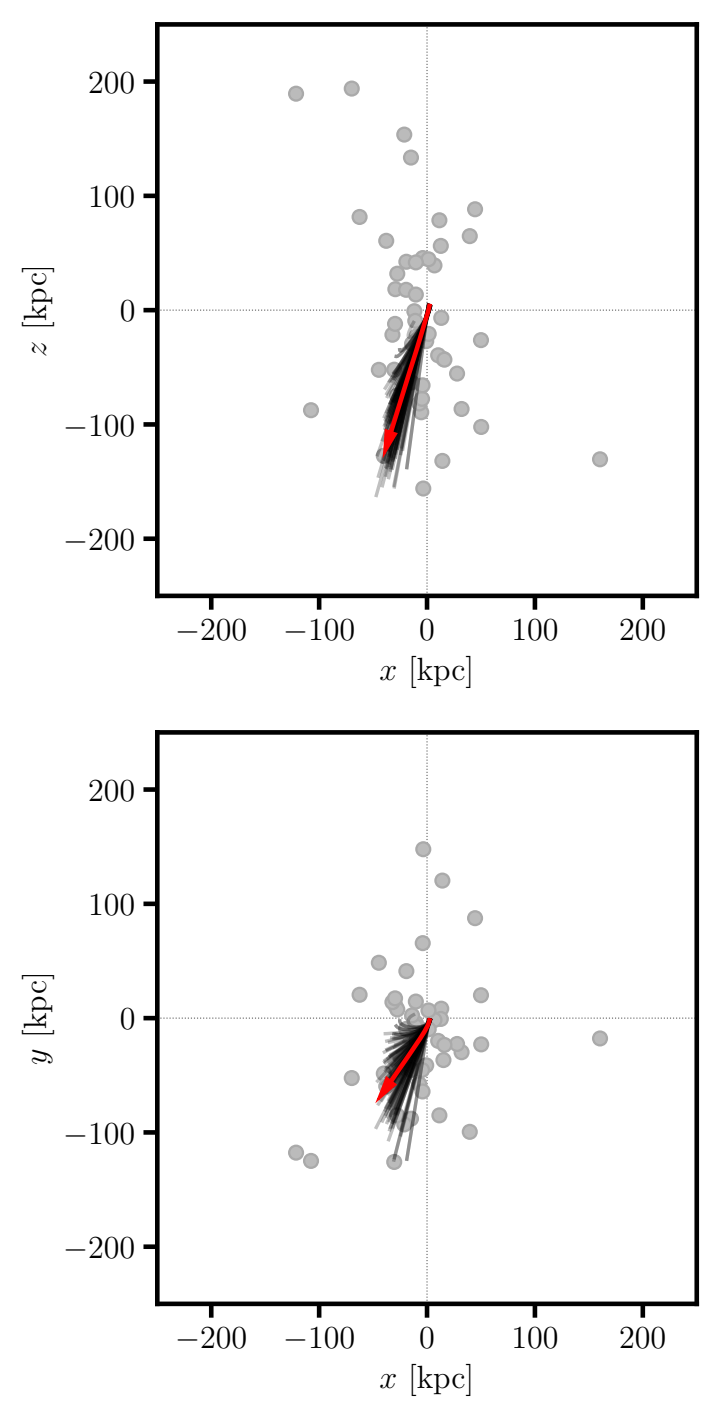

Figure 2. Integrated orbits of 2MASS J151113.24-213003.0 over 10 Gyr from 1,000 Monte Carlo realizations in a Milky Way-like potential. We plot individual orbits as gray lines and the maximum a posteriori orbit as the red arrow. We also indicate the positions of 49 classical and ultra-faint Milky Way dwarf galaxies as gray points. The maximum a posteriori orbit is aligned with a plane passing through most of the Milky Way's satellite galaxies. While most realizations result in unbound orbits, some remain bound.

$[\mathrm{Fe} / \mathrm{H}]=-3.0 \pm 0.5$. We refer to this distance posterior as "uninformed". The net effect is that our default "informed" analysis places 2MASS J151113.24-213003.0 about $3 \mathrm{kpc}$ further away from the Sun than the "uniformed" analysis would suggest (a 2.5- $\sigma$ difference). Using this new distance posterior, we integrated orbits for 10 Gyr using the same Milky Way-like potential. The eccentricity drops from $0.93_{-0.02}^{+0.01}$ in the informed analysis to a less precise but still significantly non-zero value of 
$0.53_{-0.14}^{+0.24}$. The change in heliocentric distance between the two analyses makes 2MASS J151113.24-213003.0 more likely to be bound in the uninformed analysis, with significant differences in both $z_{\max }$ and $r_{\text {apo }}$. Pericenter and Galactocentric distance remain unchanged, and in both analyses 2MASS J151113.24-213003.0 appears to be approaching pericenter on an eccentric orbit. We present the inferred orbital parameters from both analyses in Table 1.

\section{DISCUSSION}

Our measurement of $[\mathrm{Sr}, \mathrm{Ba} / \mathrm{H}] \approx-6$ for $2 \mathrm{MASS}$ J151113.24-213003.0 is surpassed by only three ultrametal poor halo stars: BPS CS 22968-0014, BPS CS 22885-0096, and SMSS J031300.36-670839.3 (Roederer et al. 2014; Keller et al. 2014; Bessell et al. 2015; Nordlander et al. 2017). The $[\mathrm{Sr}, \mathrm{Ba} / \mathrm{Fe}] \approx-3$ abundances we measured in 2MASS J151113.24-213003.0 are more than a factor of two smaller than any previous measurement for an extremely metal-poor star in the comprehensive Stellar Abundances for Galactic Archaeology (SAGA) Database (Suda et al. 2008). Despite its recordlow neutron-capture abundances, 2MASS J151113.24213003.0 has $[\mathrm{Sr} / \mathrm{Ba}]=-0.11 \pm 0.14$ and is therefore fully consistent with the $[\mathrm{Sr} / \mathrm{Ba}]=-0.25$ inferred for the solar $r$-process (e.g., Sneden et al. 2008). We also showed that 2MASS J151113.24-213003.0 is on an orbit coincident with the locations of numerous classical and ultra-faint dwarf galaxies. We expand on these observations and discuss their implications in the following subsections.

\subsection{Chemical Abundances of 2MASS J151113.24-213003.0}

The light, $\alpha$, and iron-peak element (i.e., $Z \leq 30$ ) chemical abundance pattern of 2MASS J151113.24213003.0 is consistent with the abundance ratios observed in metal-poor giants in the Milky Way (Figure 3). We see the largest discrepancies in the $\alpha$ elements calcium and titanium, where we find that 2MASS J151113.24-213003.0 has [Ca, Ti/Fe] 0. However, two other $\alpha$ elements magnesium and silicon are higher than the Roederer et al. (2014) field population $([\mathrm{Mg} / \mathrm{Fe}]=0.55$ and $[\mathrm{Si} / \mathrm{Fe}]=0.66)$, producing an average $[\langle\mathrm{Mg}, \mathrm{Ca}, \mathrm{Si}, \mathrm{Ti}\rangle / \mathrm{Fe}]$ value of 0.35 . We note that because 2MASS J151113.24-213003.0 is a relatively cool giant, it is probably more affected by non-LTE effects than most stars in the Roederer et al. (2014) sample. For this reason, we expect that some of elemental abundances of 2MASS J151113.24-213003.0 will lie near the edge of the Roederer et al. (2014) abundance distribution. This effect was clearly seen by Casey \& Schlaufman
(2015) for manganese in low surface gravity extremely metal-poor giants analyzed in a similar way.

In contrast to the light, $\alpha$, and iron-peak element abundance pattern, we find that the neutron-capture element abundance pattern in 2MASS J151113.24213003.0 differs significantly from the Milky Way's halo population. This is obvious in Figure 4, where we compare the spectra of 2MASS J151113.24-213003.0 and HD 126587-another extremely metal-poor giant with similar stellar parameters - in the vicinity of the strongest Sr II and Ba II transitions. These transitions are usually very strong in metal-poor giants. However, in the spectrum of 2MASS J151113.24-213003.0 we find the equivalent width of the Ba II line at $455 \mathrm{~nm}$ is only $5 \mathrm{~m} \AA .{ }^{6}$ Similarly, we estimate that the equivalent widths of the Sr II lines at $408 \mathrm{~nm}$ and $422 \mathrm{~nm}$ to be about 15 $\mathrm{m} \AA$. We note that hidden blends may be contributing up to $9 \mathrm{~m} \AA$ to the $422 \mathrm{~nm}$ line, as the ratio of oscillator strengths between these two Sr II transitions indicates that the equivalent width of the $408 \mathrm{~nm}$ line should be about twice as large than the $422 \mathrm{~nm}$ transition. In any case, the weaknesses of these lines imply extremely low abundances of strontium and barium: $[\mathrm{Sr}, \mathrm{Ba} / \mathrm{H}] \approx-6$ and $[\mathrm{Sr}, \mathrm{Ba} / \mathrm{Fe}] \approx-3$.

These extraordinary abundances are a significant departure from the typical neutron-capture abundances seen in metal-poor giants in the halo of the Milky Way, even after accounting for the large intrinsic scatter seen in $[\mathrm{Sr} / \mathrm{Fe}]$ and $[\mathrm{Ba} / \mathrm{Fe}]$. More quantitatively, if we represent the abundance ratios of $[\mathrm{Ba} / \mathrm{Fe}]$ seen in metal-poor giants in the halo of the Milky Way by a Gaussian with parameters $\mu=-0.57$ and $\sigma=0.44$ dex (e.g., Yong et al. 2013), then 2MASS J151113.24-213003.0 sits more than $5 \sigma$ below the mean value. We find the same result for $[\mathrm{Sr} / \mathrm{Fe}]$, but we note that the two abundance ratios are correlated to some degree.

It is well known that the neutron-capture elements in the Sun are produced in near equal proportion through the $r$ - and $s$-processes (e.g., Sneden et al. 2008). The $s$-process elements in the Sun are thought to have been created in intermediate-mass AGB stars. On the other hand, due to the Gyr timescale required for an intermediate-mass star to reach the AGB, there is no evidence for $s$-process contribution to the neutroncapture abundances of stars with $[\mathrm{Fe} / \mathrm{H}] \lesssim-2.8$ (e.g., Simmerer et al. 2004). While there are clearly exceptions for stars in binary systems where mass transfer appears to have occurred, this is unlikely to be the case for 2MASS J151113.24-213003.0 given its low

\footnotetext{
${ }^{6}$ We determined the Ba II abundance by spectral synthesis. We include this equivalent width measurement for completeness.
} 


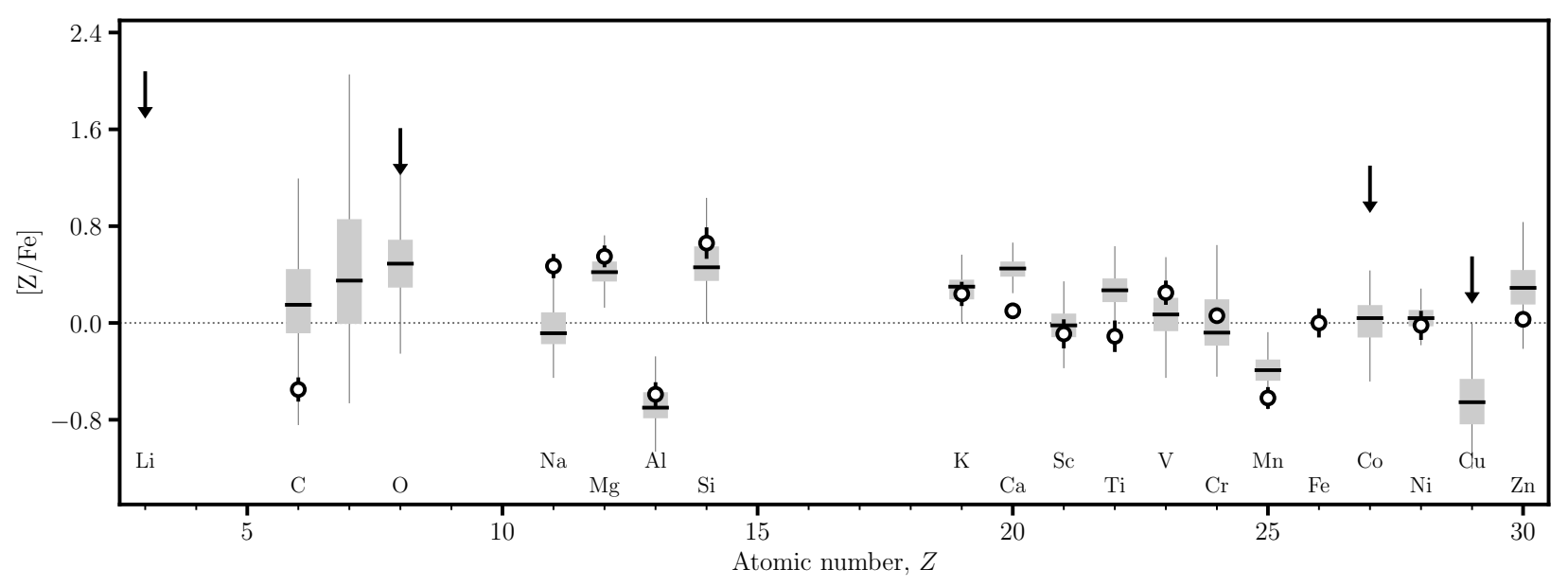

Figure 3. The light, $\alpha$, and iron-peak element abundance ratios of 2MASS J151113.24-213003.0 (circles and upper limits) compared to the distribution of abundance ratios from Roederer et al. (2014) for metal-poor field giants (boxes and whiskers). In general, 2MASS J151113.24-213003.0 shows element abundance ratios that are consistent with other metal-poor field stars for $Z \leq 30$.

neutron-capture abundances and subsolar $[\mathrm{C} / \mathrm{Fe}]$ ratio. As a result, the neutron-capture elements in 2MASS J151113.24-213003.0 are unlikely to have been produced in the standard $s$-process.

More exotic models of the $s$-process may be possible in the early Universe. Cescutti et al. (2013) used models of the $s$-process in massive, fast-rotating, and metalpoor stars by Frischknecht et al. $(2012,2016)$ to suggest that these "spinstars" could be responsible for some of the scatter in the $[\mathrm{Sr} / \mathrm{Ba}]$ ratio observed in stars with $[\mathrm{Fe} / \mathrm{H}] \lesssim-2.5$. While the Frischknecht et al. (2012, 2016) model of the $s$-process in a $M_{*}=25 M_{\odot}$ star with $[\mathrm{Fe} / \mathrm{H}]=-7$ can explain the $[\mathrm{Sr} / \mathrm{Ba}]=-0.11$ ratio we observe in 2MASS J151113.24-213003.0, those same models predict $[\mathrm{C} / \mathrm{Fe}] \approx 1.2$ and $[\mathrm{O} / \mathrm{Fe}] \approx 1.4$ that are excluded by our observations. Consequently, the neutron-capture elements in 2MASS J151113.24213003.0 are unlikely to have been produced by an exotic $s$-process in a spinstar.

\subsection{A Disrupted Dwarf Galaxy Star?}

The $r$-process-enhanced stars in Reticulum II not withstanding, it appears that many metal-poor giants in dwarf galaxies have low abundances of the neutroncapture elements (e.g., Frebel \& Norris 2015). To put the abundances of 2MASS J151113.24-213003.0 in context, we plot in Figure 5 its $[\mathrm{Sr} / \mathrm{Fe}]$ and $[\mathrm{Ba} / \mathrm{Fe}]$ abundance ratios along with a sample of both Milky Way halo and dwarf galaxy giant stars (Fulbright et al. 2004; Koch et al. 2008, 2013; Cohen \& Huang 2009, 2010; Frebel et al. 2010, 2014; Norris et al. 2010; Simon et al. 2010; Tafelmeyer et al. 2010; Honda et al. 2011; Kirby \& Cohen 2012; Gilmore et al. 2013; Ishigaki et al. 2014; Jablonka et al. 2015; Simon et al. 2015a; François et al. 2016; Ji et al. 2016a,b,c; Kirby et al. 2017). Our measurement of $[\mathrm{Sr}, \mathrm{Ba} / \mathrm{H}] \approx-6$ for $2 \mathrm{MASS}$ J151113.24-213003.0 is lower than any reported measurements for dwarf galaxy members. Our measurement of $[\mathrm{Sr}, \mathrm{Ba} / \mathrm{Fe}] \approx-3$ is surpassed only for strontium by SDSS J100710.07+160623.9, a star with $[\mathrm{Fe} / \mathrm{H}]=-1.66$ in the UFD galaxy Segue 1.

The extraordinarily-low neutron capture abundances of 2MASS J151113.24-213003.0 and the apparent overabundance of such stars in dwarf galaxies relative to the halo begs the question: did 2MASS J151113.24213003.0 form in an undiscovered or now-disrupted dwarf galaxy? If 2MASS J151113.24-213003.0 formed in a dwarf galaxy, then regardless of the fate of its parent system the orbital properties of 2MASS J151113.24213003.0 should be very similar to those of its parent system. In Figure 2 we plotted possible orbits of $2 \mathrm{MASS}$ J151113.24-213003.0 consistent with the best available observational data as well as the positions of 49 classical or ultra-faint dwarf galaxies that orbit the Milky Way. The known dwarf galaxies are arrayed in a plane that is nearly coincident with the orbital plane of 2MASS J151113.24-213003.0 (e.g., Pawlowski et al. 2012). The alignment between its orbit and the disk of satellites is a hint that 2MASS J151113.24-213003.0 may have formed in a galaxy with a similar orbit.

Cosmological simulations preformed by Wetzel (2011) suggest that some dwarf galaxies will be accreted by Milky Way-like systems on radial orbits with high eccentricities $\langle e\rangle \gtrsim 0.85$. Those dwarf galaxies on radial orbits are more likely to experience tidal stripping near pericenter and be disrupted (e.g., Dekel et al. 2003; 

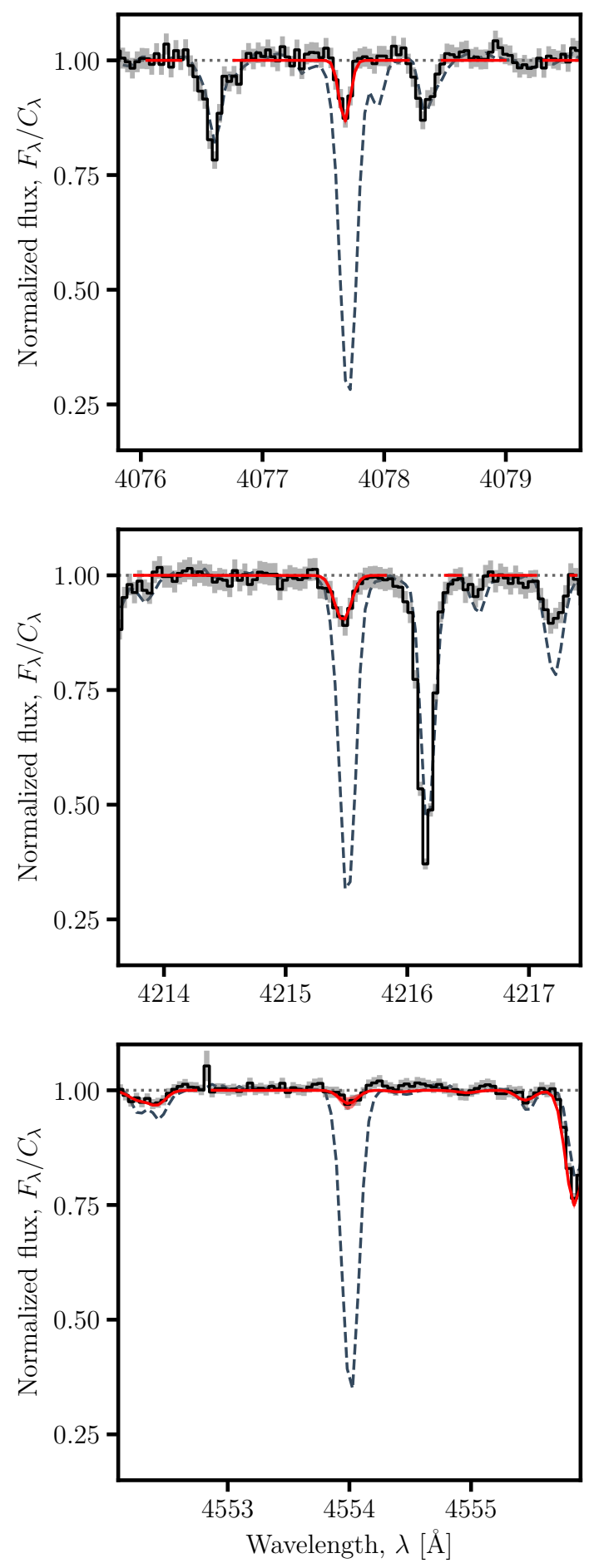

Figure 4. Continuum-normalized Magellan/MIKE spectrum surrounding the Sr II and Ba II lines measurable in 2MASS J151113.24-213003.0. We plot the observed spectrum in black, its uncertainty in gray, and the optimized model in red. For comparison we also plot with the dashed line the normalized spectrum of HD 126587, an extremely metal-poor giant with similar stellar parameters but with solar abundance ratios of strontium and barium.

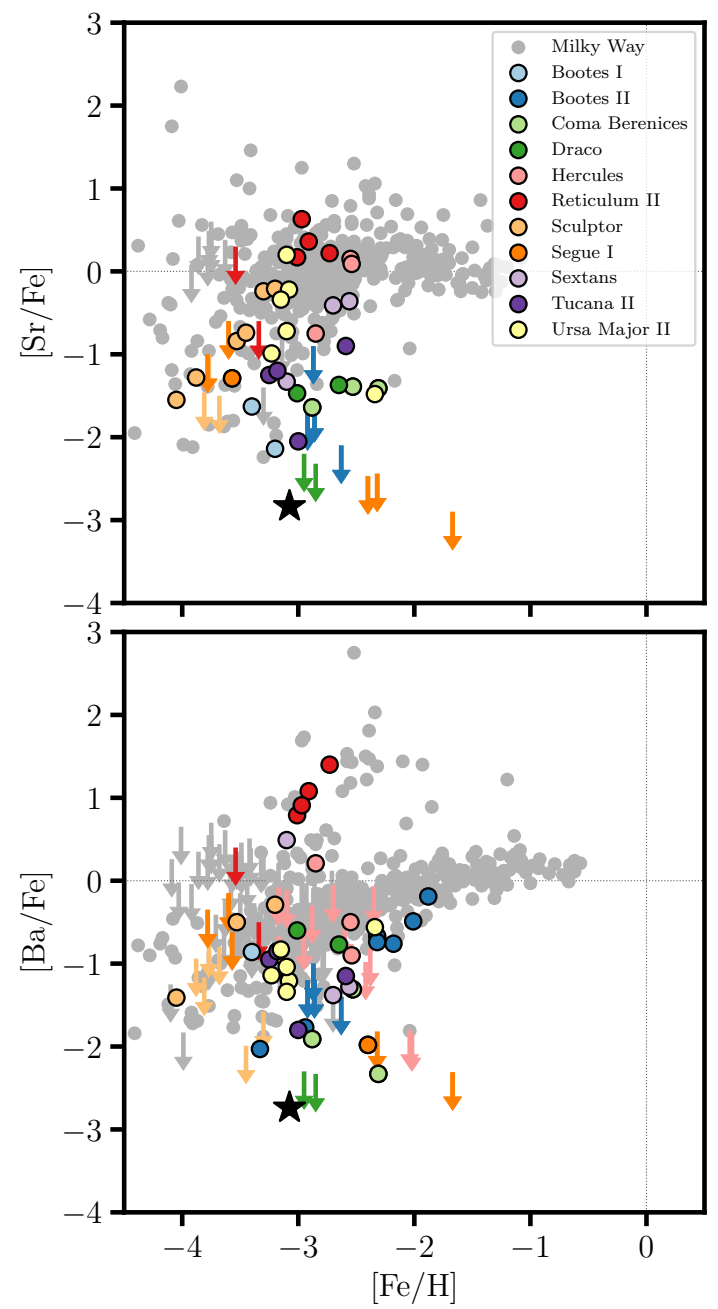

Figure 5. Strontium and barium abundance ratios of giant stars in the Milky Way field and dwarf galaxies. We indicate the position of 2MASS J151113.24-213003.0 with a black star in both panels. We plot Milky Way giants from the large samples of François et al. (2007), Ishigaki et al. (2013), Yong et al. (2013), and Roederer et al. (2014) as gray points. We plot stars in classical and ultra-faint dwarf galaxies as colored points or arrows for upper limits. 2MASS J151113.24-213003.0 has significantly lower neutron-capture abundances than any star previously known.

Taylor \& Babul 2004; McCarthy et al. 2008). Intriguingly, 2MASS J151113.24-213003.0 is on such an orbit. 2MASS J151113.24-213003.0 remains on a highlyeccentric orbit even in our uninformed analysis with extremely conservative uncertainties on its spectroscopic properties.

The small number of giant stars observed in lowmass dwarf galaxies in the halo implies that any tidallydisrupting low-mass dwarf galaxy at $\sim 10 \mathrm{kpc}$ would have an extremely low surface brightness and be distributed 
over many tens of square degrees. Pan-STARRS provides the best imaging data along the line of sight to 2MASS J151113.24-213003.0, but we see no evidence of a dwarf galaxy or surface brightness substructure in that part of the sky. However, given the possibility that the parent system could be very dispersed, the absence of evidence for low-mass dwarf galaxy or surface brightness substructure is not evidence of absence.

While the abundances and orbit of 2MASS J151113.24213003.0 provide circumstantial evidence of its possible origin in a now-disrupted dwarf galaxy, it is impossible to firmly make that association with those data alone. To explore the possible association further, we searched the SAGA database for other metal-poor giants with $[\mathrm{Fe} / \mathrm{H}] \lesssim-3.0, \log g \lesssim 3.0$, and $[\mathrm{Sr}, \mathrm{Ba} / \mathrm{Fe}] \lesssim-1.5$. We cross matched those stars with the UCAC5 proper motion database and retained those stars with $5-\sigma$ proper motion detections. The five stars so selected were BPS CS 22885-0096 and BPS CS 22960-0048 from Roederer et al. (2014) along with BPS CS 22968-0014, BPS CS 29502-0042, and BPS CS 30325-0094 from François et al. (2007). We estimated the distance to each star and calculated their orbits using radial velocities from Bonifacio et al. (2009) and Roederer et al. (2014) following the same approach that we described in Section 3.3. We find that all five stars save BPS CS 295020042 have $r_{\text {apo }} \gtrsim 20 \mathrm{kpc}$ and are therefore members of the outer halo. Assuming the $r_{\text {apo }}$ distribution from Beers et al. (2017), the probability that four of five randomly-selected extremely metal-poor field stars have $r_{\text {apo }} \gtrsim 20 \mathrm{kpc}$ is only $3 \%$ (about $1.9 \sigma$ ). The outer halo is known to have a significantly larger fraction of its stellar population contributed by disrupted dwarf galaxies than the inner halo (e.g., Carollo et al. 2007; Schlaufman et al. 2012), so the statistical association of the neutron-capture poor phenomenon with the outer halo also points to its association with dwarf galaxies.

We conclude this subsection by summarizing three relevant facts. First, 2MASS J151113.24-213003.0 is on an eccentric orbit with apocenter $\gtrsim 100 \mathrm{kpc}$ aligned with the disk of satellites in the halo of the Milky Way. Second, neutron-capture poor giants appear to be more common in dwarf galaxies than in the field halo population. Third, neutron-capture poor stars in the halo tend to have large apocenters. While individually none of these observations are conclusive proof of 2MASS J151113.24213003.0's origin in a now-disrupted dwarf galaxy, we argue that the preponderance of evidence points in that direction. If 2MASS J151113.24-213003.0 did originate in a dwarf galaxy, then it would be much closer and brighter than any other star formed in a dwarf galaxy. Thanks to their proximity and therefore bright appar- ent magnitudes, 2MASS J151113.24-213003.0 and the other neutron-capture poor extremely metal-poor giants potentially provide the best opportunity to study the origin of the heaviest elements in dwarf galaxies.

\subsection{Origin of the r-process material in 2MASS J151113.24-213003.0}

2MASS J151113.24-213003.0 has extraordinarily-low neutron capture abundances $[\mathrm{Sr}, \mathrm{Ba} / \mathrm{H}] \approx-6.0$ and $[\mathrm{Sr}, \mathrm{Ba} / \mathrm{Fe}] \approx-3.0$, but solar $[\mathrm{Sr} / \mathrm{Ba}] \approx-0.1$. Any possible origin for its neutron-capture elements must occur promptly and be able to simultaneously explain both observations. We have shown that the neutroncapture elements in 2MASS J151113.24-213003.0 cannot be attributed to a standard or exotic $s$-process, so they must have been created by the $r$-process. While the origin of the $r$-process has been thoroughly debated since it was first described by Burbidge et al. (1957) and Cameron (1957a,b), we will limit our discussion to the two sites most favored today: core-collapse supernovae and neutron-star mergers.

For most of its history, core-collapse supernovae have been the favored astrophysical origin of the $r$-process. Many models in the literature can produce neutroncapture material following a core-collapse supernovae, and most rely on a neutrino-driven wind expected to be produced in the first few seconds following the creation of a neutron star. In the high temperature and entropy conditions in the evacuated bubble surrounding the newly-formed neutron star, a neutrino can bind with a proton to produce a neutron and a positron, driving an expanding wind and producing a bubble of neutronrich material to seed the $r$-process (e.g., Meyer et al. 1992). However, the $r$-process yields in this scenario are extremely sensitive to the equation of state, the electron fraction, the neutrino properties, and the $\beta$-decay rate, among other factors (e.g., Qian \& Woosley 1996; Hoffman et al. 1997; Thompson et al. 2001).

Most models find that neutrino-driven winds from a proto-neutron star following a core-collapse supernova can be very effective at producing less-massive $r$-process nuclei (e.g., strontium, yttrium, and zirconium). In contrast, it is generally difficult to produce heavier nuclei with $A \gtrsim 130$ like barium, and this under production of heavy nuclei relative to the solar $r$-process pattern has remained a problem (e.g., Roberts et al. 2010; Roberts 2012). Some groups have succeeded in producing solar ratios of $r$-process material following core-collapse supernovae, but these successes critically depend on a sensitive time-dependent electron fraction and other uncertain factors. They also require strong magnetic fields or neutron star masses in excess of $2 M_{\odot}$ to create the 
heavier nuclei in significant proportions (e.g., Wanajo 2013). While neutron star masses near $2 M_{\odot}$ have been inferred in the field (e.g., Lattimer 2012), the causality limit breaks above $2.4 M_{\odot}$. In short, most models of core-collapse supernovae generally have trouble producing heavy $r$-process elements like barium in the solar $r$-process ratio unless somewhat exotic conditions are invoked.

Population III stars with $140 M_{\odot} \lesssim M_{*} \lesssim 260 M_{\odot}$ could end their lives as pair-instability supernovae and may produce neutron-capture elements in some situations. If the neutron-capture elements in 2MASS J151113.24-213003.0 were the result of a pair-instability supernova, then Heger \& Woosley (2002) predicted that we should observe a strong odd/even effect in the light and $\alpha$ elements as well as very little zinc. We do not see an odd/even effect and we see $[\mathrm{Zn} / \mathrm{Fe}]=0.03$. Accordingly, the neutron-capture elements in 2MASS J151113.24-213003.0 are unlikely to have been produced by the $r$-process in a pair-instability supernovae.

Nevertheless, core-collapse supernovae are an attractive astrophysical site for the creation of the neutroncapture elements in 2MASS J151113.24-213003.0. Supernovae must have occurred before the formation of 2MASS J151113.24-213003.0 as its light, $\alpha$, and ironpeak element abundances require them. Supernovae also occur promptly, so there is no timescale problem. If supernovae produce $r$-process elements, then they produce them only in small quantities - less than $10^{-7} M_{\odot}$ (e.g., Tsujimoto et al. 2000). An event that promptly produces light, $\alpha$, iron peak, and a small amount of neutron capture elements is fully consistent with the $5 \times 10^{-14} M_{\odot}$ and $2 \times 10^{-14} M_{\odot}$ of strontium and barium in 2MASS J151113.24-213003.0. Indeed, the models of Heger \& Woosley (2010) indicate that Pop III supernovae in the mass range $40 M_{\odot} \lesssim M_{*} \lesssim 75 M_{\odot}$ with a range of energies, piston locations, and mixing parameters can accommodate the abundances of 2MASS J151113.24-213003.0.

Neutron-star or black hole-neutron star mergers have attracted significant recent attention as a possible astrophysical site of the $r$-process (e.g., Lattimer \& Schramm 1976; Lattimer et al. 1977; Eichler et al. 1989; Davies et al. 1994; Freiburghaus et al. 1999). The escape of neutron-rich matter from the deep potential well of a neutron star during a merger produces rapid neutroncapture nucleosynthesis consistent with the main solar $r$-process. Neutron-rich material can escape the merger in unbound tidal tails or in a wind from a rotationallysupported accretion disk left behind by the merger and seed the interstellar medium with neutron-capture elements. Neutron-star or black hole-neutron star merg- ers are also thought to be capable of producing elements in all three peaks of the main solar $r$-process (e.g., Wu et al. 2016; Roberts et al. 2017; Fernández et al. 2017). Because of their expected rarity compared to core-collapse supernovae, neutron-star or black holeneutron star mergers are thought to produce significant quantities of neutron-capture material (e.g., Argast et al. 2004). Unlike supernovae, they require a significant lag time between the onset of star formation and injection of neutron-capture elements into the interstellar medium. They also do not produce significant amounts of the light or $\alpha$ elements.

The observation of $r$-process enrichment in the metalpoor giant stars in the UFD galaxy Reticulum II by Ji et al. (2016a) and Roederer et al. (2016) provides empirical evidence for a rare, prolific source of $r$-process elements as well as the nucleosynthesis produced by such an event. Because Reticulum II is so far unique in a sample of about 20 UFD galaxies, the process that created its neutron-capture elements is rare. The empirical properties of the nucleosynthesis event in Reticulum II can be inferred from Figures 5 and $6:[\mathrm{Sr} / \mathrm{Fe}] \approx 0.5$, $[\mathrm{Ba} / \mathrm{Fe}] \approx 1.0$, and $-1 \lesssim[\mathrm{Sr} / \mathrm{Ba}] \lesssim 0$. Given its isolation, Ji et al. (2016a) suggested that it would take more than 1,000 typical core-collapse supernovae to produce the mass of $r$-process elements present in Reticulum II. This is incompatible with the star formation history and metallicity distribution of the galaxy. Instead, Ji et al. (2016a) favored an event that produced a large amount of neutron-capture elements without creating much iron. They suggested that either a neutron-star merger or a magnetorotationally-driven supernova (e.g., Winteler et al. 2012) were the best candidates, though the full distribution of neutron-capture material in Reticulum II may still require an additional source (Ji et al. 2016c).

Taking all of these facts into account, the best explanation for the origin of the neutron-capture elements in 2MASS J151113.24-213003.0 is a Pop III or extreme Pop II core-collapse supernova. Core-collapse supernovae are prompt, and at least one such explosion is necessary to have created the $Z \leq 30$ elements in 2MASS J151113.24-213003.0. Despite the difficulties theoretical models of the $r$-process in core-collapse supernovae have producing elements with $A \gtrsim 130$, our observation empirically suggests that somewhere in some core-collapse supernovae strontium and barium are both produced in the solar ratio. Rare $r$ process events - presumably mergers involving a neutron star or magnetorotationally-driven supernova-like the event that occurred in Reticulum II produce [Sr/Fe] and $[\mathrm{Ba} / \mathrm{Fe}]$ far too high to explain our observations of 2MASS J151113.24-213003.0. This conclusion is 


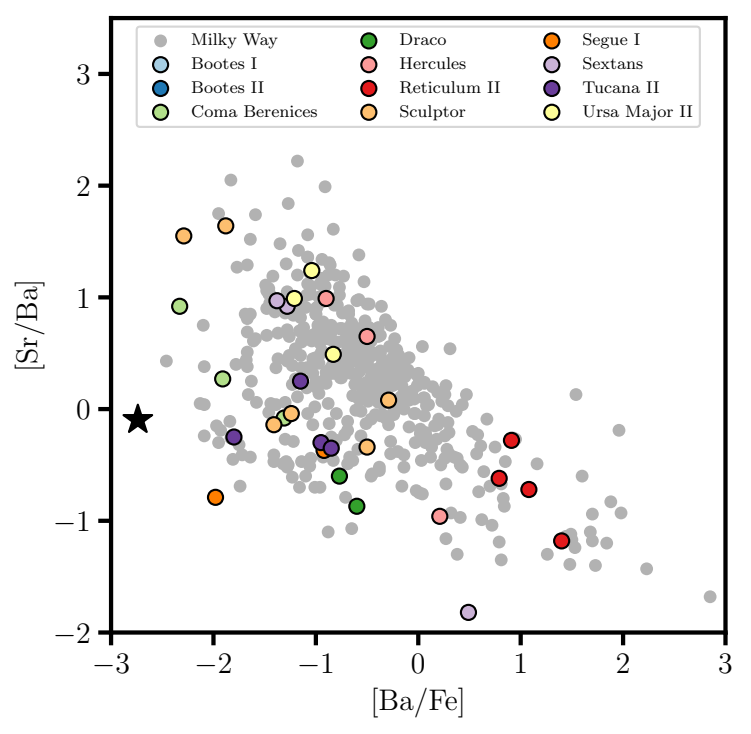

Figure 6. $[\mathrm{Ba} / \mathrm{Fe}]$ versus $[\mathrm{Sr} / \mathrm{Ba}]$ for very metal-poor giants omitting stars with only upper limits on strontium or barium. We indicate the position of 2MASS J151113.24213003.0 with a black star. We plot Milky Way giants from the large samples of François et al. (2007), Ishigaki et al. (2013), Yong et al. (2013), and Roederer et al. (2014) as gray points. We plot stars in classical and ultra-faint dwarf galaxies as colored points. 2MASS J151113.24-213003.0 has extremely low $[\mathrm{Ba} / \mathrm{Fe}]$ but solar $[\mathrm{Sr} / \mathrm{Ba}]$.

strengthened by the evidence we presented above that 2MASS J151113.24-213003.0 formed in a now-disrupted dwarf galaxy. That is, Reticulum II demonstrates the abundance signatures of stars in dwarf galaxies seeded with neutron-capture elements by a rare, prolific event. The fact that 2MASS J151113.24-213003.0 does not fit that pattern suggests its neutron-capture elements were created by a qualitatively different process.

While 2MASS J151113.24-213003.0 is superlative in that it has the lowest $[\mathrm{Sr} / \mathrm{Fe}]$ and $[\mathrm{Ba} / \mathrm{Fe}]$ yet seen, the most neutron-capture poor stars previously known share many of its properties. François et al. (2007) and Lai et al. (2008) found that extremely metal-poor stars in the Milky Way with decreasing $[\mathrm{Ba} / \mathrm{H}]$ abundance ratios showed higher $[\mathrm{Sr} / \mathrm{Ba}]$ representative of a possible weak $r$-process in contrast the solar $[\mathrm{Sr} / \mathrm{Ba}]$ expected in the main $r$-process. In Figure 7, we plot the abundances of 2MASS J151113.24-213003.0 along with the empirical yields of both the main and weak $r$-process. We follow Roederer (2017) and define the weak $r$-process as the average abundances of the stars HD 88609 and HD 122563 from Honda et al. (2006, 2007). We define the main $r$-process as the average abundances of the stars BPS CS 22892-0052 and BPS CS 31082-0001 from Sneden et al. (2003, 2009) and Hill et al. (2002). We extend to $[\mathrm{Ba} / \mathrm{H}] \lesssim-6$ the results of François et al. (2007) and Lai et al. (2008) that for neutron-capture poor stars with $[\mathrm{Ba} / \mathrm{H}] \lesssim-4.5$ the main $r$-process is favored over the weak $r$-process preferred for common extremely metal-poor stars. François et al. (2007) went on to suggest different regimes where weak $r$-process models could play a role before concluding that it was unnecessary for stars with $[\mathrm{Ba} / \mathrm{H}] \lesssim-4.5$. As a result, we assert that the $r$-process material in 2MASS J151113.24-213003.0 is consistent with being produced wholly from the main $r$-process with no contributions from the weak $r$-process.

These observations extend the parameter space in which the $r$-process appears to be universal. The universality of the $r$-process usually refers to the idea that the neutron-capture abundance pattern in $r$-process enhanced metal-poor stars is very similar to the inferred solar $r$-process pattern describes. This remarkable observation implies that the same $r$-process pattern observed in the Sun is seen in both extraordinarily neutroncapture rich and extraordinarily neutron-capture poor extremely metal-poor stars.

The extraordinarily low but non-zero abundances of neutron-capture elements we observed in 2MASS J151113.24-213003.0 and our conclusion that Pop III or extreme Pop II supernovae produced neutron-capture elements leads to the prediction that there will never be a Pop II star found without any neutron-capture elements. Our inference that 2MASS J151113.24-213003.0 formed in a now-disrupted dwarf galaxy suggests that stars in dwarf galaxies with low upper limits on the their strontium and barium abundances have comparable abundances to those we see in 2MASS J151113.24213003.0 and are not neutron-capture free. We predict that when high-resolution spectrographs on $30 \mathrm{~m}$ class telescopes are used to investigate the neutron-capture abundances of stars in dwarf galaxies that are currently too faint for high-resolution spectroscopy, they will not find any stars without neutron-capture elements. We suggest that the same will be true in the large number of as-of-yet undiscovered UFD galaxies that will be found by the Large Synoptic Survey Telescope.

\section{CONCLUSIONS}

We described the properties of 2MASS J151113.24213003.0, a star we discovered and found to have $[\mathrm{Sr}, \mathrm{Ba} / \mathrm{H}] \approx-6.0$ and $[\mathrm{Sr}, \mathrm{Ba} / \mathrm{Fe}] \approx-3.0$. These are the lowest abundances of strontium and barium relative to iron ever observed. In addition, the star has $[\mathrm{Sr} / \mathrm{Ba}]=-0.11 \pm 0.14$ consistent with the scaled solar $r$-process pattern that has $[\mathrm{Sr} / \mathrm{Ba}]=-0.25$. We 


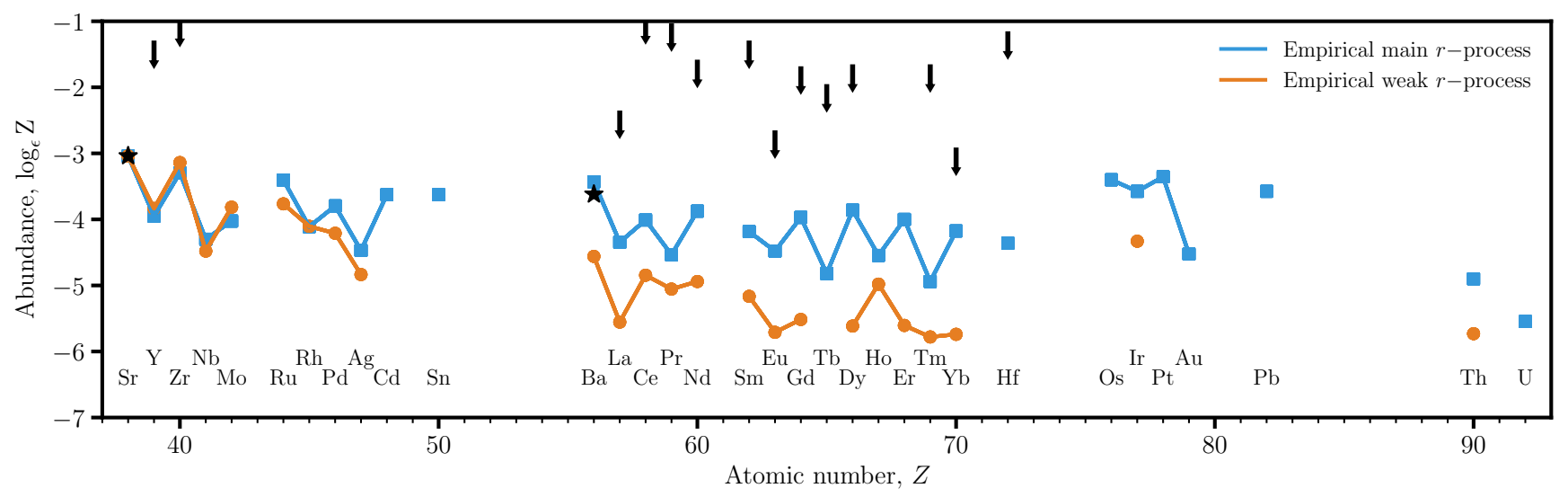

Figure 7. Neutron-capture element abundances and upper limits for 2MASS J151113.24-213003.0. We also plot the empirical yields of the main and weak $r$-processes in blue and orange respectively. The $y$ axis has been clipped for clarity and not all upper limits are shown. We have scaled both theoretical curves to the [Sr/H] abundance of 2MASS J151113.24-213003.0. The [Ba/Fe] ratio of 2MASS J151113.24-213003.0 indicates that its neutron-capture abundances are consistent with the main $r$-process.

find that 2MASS J151113.24-213003.0 has an extreme orbit taking it beyond $100 \mathrm{kpc}$ that is aligned with the disk of satellites in the halo of the Milky Way. These observations indicate that it may have formed in a now-disrupted dwarf galaxy. We confirm that other neutron-capture poor stars preferentially have Galactic orbits with apocenters beyond $20 \mathrm{kpc}$, suggesting that neutron-capture poor stars belong to the outer halo stellar population. Since the contribution of disrupted dwarf galaxies to the halo increases significantly with distance and many stars in surviving dwarf galaxies are neutron-capture poor, this relationship supports the idea that neutron-capture poor stars form in dwarf galaxies. After considering both the standard and exotic $s$-process as well as the $r$-process in core-collapse supernovae, pair instability supernovae, and mergers involving a neutron star we concluded that the explosion of a Pop III or extreme Pop II star provided the best explanation for the origin of the neutron-capture elements in 2MASS J151113.24-213003.0. Despite the apparent theoretical difficulty in doing so, this empirical inference requires that both strontium and barium be formed in the solar ratio somewhere in some kind of core-collapse supernovae.

We thank the anonymous referee for a prompt, detailed, and insightful review. We thank Prashin Jethwa (Cambridge) for assistance with observations. We thank Denis Erkal (Cambridge), Brendan Griffen (Scaled Biolabs), Alex Ji (Carnegie Observatories), Andy Lipnicky (Rochester IoT), Andy McWilliam (Carnegie Observatories), Sergey Koposov (Carnegie Mellon), David Nataf (Johns Hopkins), Adrian Price-Whelan (Prince- ton) and Luke Roberts (Michigan State) for valuable discussions. A. R. C. acknowledges support from the Australian Research Council through Discovery Project grant DP160100637. This research has made use of: Astropy, a community-developed core Python package for Astronomy (Astropy Collaboration et al. 2013); NASA's Astrophysics Data System Bibliographic Services; and the SIMBAD database and VizieR catalog access tools provided by CDS, Strasbourg, France. The original description of the VizieR service was published by Ochsenbein et al. (2000). This publication makes use of data products from the Two Micron All Sky Survey, which is a joint project of the University of Massachusetts and the Infrared Processing and Analysis Center/California Institute of Technology, funded by the National Aeronautics and Space Administration and the National Science Foundation. This publication makes use of data products from the Wide-field Infrared Survey Explorer, which is a joint project of the University of California, Los Angeles, and the Jet Propulsion Laboratory/California Institute of Technology, funded by the National Aeronautics and Space Administration. This research was made possible through the use of the AAVSO Photometric All-Sky Survey (APASS), funded by the Robert Martin Ayers Sciences Fund.

Facilities: Magellan:Clay (MIKE echelle spectrograph)

Software: astropy (Astropy Collaboration et al. 2013), CarPy (Kelson 2003; Kelson et al. 2014), galpy (Bovy 2015), isochrones (Morton 2015), matplotlib (Hunter et al. 2007), MOOG (Sneden 1973), numpy (van der Walt et al. 2011), scipy (Oliphant et al. 2001) 


\section{REFERENCES}

Altmann, M., Roeser, S., Demleitner, M., Bastian, U., \& Schilbach, E. 2017, A\&A, 600, L4

Argast, D., Samland, M., Thielemann, F.-K., \& Qian, Y.-Z. 2004, A\&A, 416, 997

Astropy Collaboration, Robitaille, T. P., Tollerud, E. J., et al. 2013, A\&A, 558, A33

Barklem, P. S., Belyaev, A. K., \& Asplund, M. 2003, A\&A, 409, L1

Barklem, P. S., Stempels, H. C., Allende Prieto, C., et al. 2002, A\&A, 385, 951

Bechtol, K., Drlica-Wagner, A., Balbinot, E., et al. 2015, ApJ, 807, 50

Beers, T. C., Placco, V. M., Carollo, D., et al. 2017, ApJ, 835,81

Bernstein, R., Shectman, S. A., Gunnels, S. M., Mochnacki, S., \& Athey, A. E. 2003, Proc. SPIE, 4841, 1694

Bessell, M. S., Collet, R., Keller, S. C., et al. 2015, ApJL, 806, L16

Biémont, E., Palmeri, P., \& Quinet, P. 1999, Ap\&SS, 269, 635

Bonifacio, P., Spite, M., Cayrel, R., et al. 2009, A\&A, 501, 519

Bovy, J. 2015, ApJS, 216, 29

Bovy, J., Allende Prieto, C., Beers, T. C., et al. 2012, ApJ, 759,131

Burbidge, E. M., Burbidge, G. R., Fowler, W. A., \& Hoyle, F. 1957, Reviews of Modern Physics, 29, 547

Cameron, A. G. W. 1957a, AJ, 62, 9

Cameron, A. G. W. 1957b, PASP, 69, 201

Carollo, D., Beers, T. C., Lee, Y. S., et al. 2007, Nature, 450, 1020

Casey, A. R. 2014, Ph.D. Thesis,

Casey, A. R., \& Schlaufman, K. C. 2015, ApJ, 809, 110

Castelli, F., \& Kurucz, R. L. 2004, arXiv:astro-ph/0405087

Cayrel, R., Depagne, E., Spite, M., et al. 2004, A\&A, 416, 1117

Cescutti, G., Chiappini, C., Hirschi, R., Meynet, G., \& Frischknecht, U. 2013, A\&A, 553, A51

Choi, J., Dotter, A., Conroy, C., et al. 2016, ApJ, 823, 102

Cohen, J. G., Christlieb, N., Thompson, I., et al. 2013, ApJ, 778, 56

Cohen, J. G., \& Huang, W. 2009, ApJ, 701, 1053

Cohen, J. G., \& Huang, W. 2010, ApJ, 719, 931

Davies, M. B., Benz, W., Piran, T., \& Thielemann, F. K. 1994, ApJ, 431, 742

Dekel, A., Devor, J., \& Hetzroni, G. 2003, MNRAS, 341, 326

Dotter, A. 2016, ApJS, 222, 8
Dotter, A., Chaboyer, B., Jevremović, D., et al. 2008, ApJS, 178, 89

Eichler, D., Livio, M., Piran, T., \& Schramm, D. N. 1989, Nature, 340, 126

Fernández, R., Foucart, F., Kasen, D., et al. 2017, Classical and Quantum Gravity, 34, 154001

Fuhrmann, K. 1998, A\&A, 338, 161

Fulbright, J. P., Rich, R. M., \& Castro, S. 2004, ApJ, 612, 447

François, P., Depagne, E., Hill, V., et al. 2007, A\&A, 476, 935

François, P., Monaco, L., Bonifacio, P., et al. 2016, A\&A, 588, A7

Frebel, A., Casey, A. R., Jacobson, H. R., \& Yu, Q. 2013a, ApJ, 769, 57

Frebel, A., Lunnan, R., Casey, A. R., et al. 2013b, ApJ, 771,39

Frebel, A., \& Norris, J. E. 2015, ARA\&A, 53, 631

Frebel, A., Simon, J. D., Geha, M., \& Willman, B. 2010, ApJ, 708, 560

Frebel, A., Simon, J. D., \& Kirby, E. N. 2014, ApJ, 786, 74

Freiburghaus, C., Rosswog, S., \& Thielemann, F.-K. 1999, ApJL, 525, L121

Frischknecht, U., Hirschi, R., Pignatari, M., et al. 2016, MNRAS, 456, 1803

Frischknecht, U., Hirschi, R., \& Thielemann, F.-K. 2012, A\&A, 538, L2

Gilmore, G., Norris, J. E., Monaco, L., et al. 2013, ApJ, 763,61

Gilroy, K. K., Sneden, C., Pilachowski, C. A., \& Cowan, J. J. 1988, ApJ, 327, 298

Girard, T. M., van Altena, W. F., Zacharias, N., et al. 2011, AJ, 142, 15

Heiter, U., Jofré, P., Gustafsson, B., et al. 2015, A\&A, 582, A49

Heger, A., \& Woosley, S. E. 2002, ApJ, 567, 532

Heger, A., \& Woosley, S. E. 2010, ApJ, 724, 341

Henden, A. A., Levine, S., Terrell, D., \& Welch, D. L. 2015, American Astronomical Society Meeting Abstracts, 225, 336.16

Hill, V., Plez, B., Cayrel, R., et al. 2002, A\&A, 387, 560

Hoffman, R. D., Woosley, S. E., \& Qian, Y.-Z. 1997, ApJ, 482,951

Honda, S., Aoki, W., Ishimaru, Y., \& Wanajo, S. 2007, ApJ, 666, 1189

Honda, S., Aoki, W., Ishimaru, Y., Wanajo, S., \& Ryan, S. G. 2006, ApJ, 643, 1180

Honda, S., Aoki, W., Arimoto, N., \& Sadakane, K. 2011, PASJ, 63, 523 
Ishigaki, M. N., Aoki, W., Arimoto, N., \& Okamoto, S. 2014, A\&A, 562, A146

Hunter, J. D., 2007, Computing in Science \& Engineering, 9,3

Ishigaki, M. N., Aoki, W., \& Chiba, M. 2013, ApJ, 771, 67

Jablonka, P., North, P., Mashonkina, L., et al. 2015, A\&A, 583, A67

Ji, A. P., Frebel, A., Chiti, A., \& Simon, J. D. 2016a, Nature, 531, 610

Ji, A. P., Frebel, A., Ezzeddine, R., \& Casey, A. R. 2016b, ApJL, 832, L3

Ji, A. P., Frebel, A., Simon, J. D., \& Chiti, A. 2016c, ApJ, 830,93

Ji, A. P., Frebel, A., Simon, J. D., \& Geha, M. 2016d, ApJ, 817,41

Keller, S. C., Bessell, M. S., Frebel, A., et al. 2014, Nature, 506, 463

Kelson, D. D. 2003, PASP, 115, 688

Kelson, D. D., Williams, R. J., Dressler, A., et al. 2014, ApJ, 783, 110

Kirby, E. N., \& Cohen, J. G. 2012, AJ, 144, 168

Kirby, E. N., Cohen, J. G., Simon, J. D., et al. 2017, ApJ, 838,83

Koch, A., McWilliam, A., Grebel, E. K.,

Koch, A., Feltzing, S., Adén, D., \& Matteucci, F. 2013, A\&A, 554, A5

Koch, A., Hansen, T., Feltzing, S., \& Wilkinson, M. I. 2014, ApJ, 780, 91 Zucker, D. B., \& Belokurov, V. 2008, ApJL, 688, L13

Koposov, S. E., Belokurov, V., Torrealba, G., \& Evans, N. W. 2015a, ApJ, 805, 130

Koposov, S. E., Casey, A. R., Belokurov, V., et al. 2015b, ApJ, 811, 62

Korn, A. J. 2002, Scientific Drivers for ESO Future VLT/VLTI Instrumentation, 199

Korn, A. J., Shi, J., \& Gehren, T. 2003, A\&A, 407, 691

Kurucz, R., \& Bell, B. 1995, Atomic Line Data

(R.L. Kurucz and B. Bell) Kurucz CD-ROM

No. 23. Cambridge, Mass.: Smithsonian Astrophysical Observatory, 1995., 23

Lai, D. K., Bolte, M., Johnson, J. A., et al. 2008, ApJ, 681, 1524-1556

Lattimer, J. M. 2012, Annual Review of Nuclear and Particle Science, 62, 485

Lattimer, J. M., Mackie, F., Ravenhall, D. G., \& Schramm, D. N. 1977, ApJ, 213, 225

Lattimer, J. M., \& Schramm, D. N. 1976, ApJ, 210, 549

Lawler, J. E., Bonvallet, G., \& Sneden, C. 2001a, ApJ, 556, 452
Lawler, J. E., Wickliffe, M. E., den Hartog, E. A., \& Sneden, C. 2001b, ApJ, 563, 1075

Lindegren, L., Lammers, U., Bastian, U., et al. 2016, A\&A, 595, A4

Masseron, T., Plez, B., Van Eck, S., et al. 2014, A\&A, 571, AA47

Matteucci, F., Romano, D., Arcones, A., Korobkin, O., \& Rosswog, S. 2014, MNRAS, 438, 2177

McCarthy, I. G., Frenk, C. S., Font, A. S., et al. 2008, MNRAS, 383, 593

McWilliam, A., Preston, G. W., Sneden, C., \& Searle, L. 1995a, AJ, 109, 2757

McWilliam, A., Preston, G. W., Sneden, C., \& Shectman, S. 1995, AJ, 109, 2736

Meyer, B. S., Mathews, G. J., Howard, W. M., Woosley, S. E., \& Hoffman, R. D. 1992, ApJ, 399, 656

Michalik, D., Lindegren, L., \& Hobbs, D. 2015, A\&A, 574, A115

Miyamoto, M., \& Nagai, R. 1975, PASJ, 27, 533

Morton, T. D. 2015, Astrophysics Source Code Library, ascl: 1503.010

Naiman, J. P., Pillepich, A., Springel, V., et al. 2017, arXiv:1707.03401

Navarro, J. F., Frenk, C. S., \& White, S. D. M. 1996, ApJ, 462,563

Nordlander, T., Amarsi, A. M., Lind, K., et al. 2017, A\&A, 597, A6

Norris, J. E., Ryan, S. G., \& Beers, T. C. 1996, ApJS, 107, 391

Norris, J. E., Peterson, R. C., \& Beers, T. C. 1993, ApJ, 415, 797

Norris, J. E., Wyse, R. F. G., Gilmore, G., et al. 2010, ApJ, 723, 1632

Ochsenbein, F., Bauer, P., \& Marcout, J. 2000, A\&AS, 143, 23

Oliphant, E., Peterson, P., et al. 2001, SciPy: Open Source Scientific Tools for Python, http://www.scipy.org/ [Online; accessed 2017-08-30]

Pawlowski, M. S., Pflamm-Altenburg, J., \& Kroupa, P. 2012, MNRAS, 423, 1109

Paxton, B., Bildsten, L., Dotter, A., et al. 2011, ApJS, 192, 3

Paxton, B., Cantiello, M., Arras, P., et al. 2013, ApJS, 208, 4

Paxton, B., Marchant, P., Schwab, J., et al. 2015, ApJS, 220, 15

Qian, Y.-Z. 2000, ApJL, 534, L67

Qian, Y.-Z., \& Woosley, S. E. 1996, ApJ, 471, 331

Roberts, L. F. 2012, ApJ, 755, 126 
Roberts, L. F., Lippuner, J., Duez, M. D., et al. 2017, MNRAS, 464, 3907

Roberts, L. F., Woosley, S. E., \& Hoffman, R. D. 2010, ApJ, 722, 954

Roederer, I. U. 2013, AJ, 145, 26

Roederer, I. U. 2017, ApJ, 835, 23

Roederer, I. U., Sneden, C., Thompson, I. B., Preston, G. W., \& Shectman, S. A. 2010, ApJ, 711, 573

Roederer, I. U., Preston, G. W., Thompson, I. B., et al. 2014, AJ, 147, 136

Roederer, I. U., \& Kirby, E. N. 2014, MNRAS, 440, 2665

Roederer, I. U., Mateo, M., Bailey, J. I., III, et al. 2016, AJ, 151, 82

Roeser, S., Demleitner, M., \& Schilbach, E. 2010, AJ, 139, 2440

Ryan, S. G., Norris, J. E., \& Beers, T. C. 1996, ApJ, 471, 254

Schlafly, E. F., \& Finkbeiner, D. P. 2011, ApJ, 737, 103

Schlaufman, K. C., \& Casey, A. R. 2014, ApJ, 797, 13

Schlaufman, K. C., Rockosi, C. M., Lee, Y. S., et al. 2012, ApJ, 749, 77

Schlegel, D. J., Finkbeiner, D. P., \& Davis, M. 1998, ApJ, 500,525

Shectman, S. A., \& Johns, M. 2003, Proc. SPIE, 4837, 910

Shen, S., Cooke, R. J., Ramirez-Ruiz, E., et al. 2015, ApJ, 807,115

Simmerer, J., Sneden, C., Cowan, J. J., et al. 2004, ApJ, 617, 1091

Simon, J. D., Frebel, A., McWilliam, A., Kirby, E. N., \& Thompson, I. B. 2010, ApJ, 716, 446

Simon, J. D., Jacobson, H. R., Frebel, A., et al. 2015a, ApJ, 802,93

Simon, J. D., Drlica-Wagner, A., Li, T. S., et al. 2015b, ApJ, 808, 95

Skrutskie, M. F., Cutri, R. M., Stiening, R., et al. 2006, AJ, 131, 1163

Sneden, C. A. 1973, PhD thesis, Univ. Texas, Austin

Sneden, C., Cowan, J. J., \& Gallino, R. 2008, ARA\&A, 46, 241

Sneden, C., Cowan, J. J., Lawler, J. E., et al. 2003, ApJ, 591,936
Sneden, C., Lawler, J. E., Cowan, J. J., Ivans, I. I., \& Den Hartog, E. A. 2009, ApJS, 182, 80

Sobeck, J. S., Kraft, R. P., Sneden, C., et al. 2011, AJ, 141, 175

Starkenburg, E., Hill, V., Tolstoy, E., et al. 2013, A\&A, 549, A 88

Suda, T., Katsuta, Y., Yamada, S., et al. 2008, PASJ, 60, 1159

Tafelmeyer, M., Jablonka, P., Hill, V., et al. 2010, A\&A, 524, A58

Taylor, J. E., \& Babul, A. 2004, MNRAS, 348, 811

Thompson, T. A., Burrows, A., \& Meyer, B. S. 2001, ApJ, 562,887

Truran, J. W., Cowan, J. J., Pilachowski, C. A., \& Sneden, C. 2002, PASP, 114, 1293

Tsujimoto, T., Shigeyama, T., \& Yoshii, Y. 2000, ApJL, 531, L33

van de Voort, F., Quataert, E., Hopkins, P. F., Kereš, D., \& Faucher-Giguère, C.-A. 2015, MNRAS, 447, 140

van der Walt, S., Colbert, C., \& Varoquaux, G., 2011, Computing in Science \& Engineering, 13, 22-30

Venn, K. A., Shetrone, M. D., Irwin, M. J., et al. 2012, ApJ, 751, 102

Walker, M. G., Mateo, M., Olszewski, E. W., et al. 2015, ApJ, 808, 108

Wanajo, S. 2013, ApJL, 770, L22

Wetzel, A. R. 2011, MNRAS, 412, 49

Winteler, C., Käppeli, R., Perego, A., et al. 2012, ApJL, 750, L22

Wright, E. L., Eisenhardt, P. R. M., Mainzer, A. K., et al. 2010, AJ, 140, 1868

Wu, M.-R., Fernández, R., Martínez-Pinedo, G., \&

Metzger, B. D. 2016, MNRAS, 463, 2323

Yong, D., Norris, J. E., Bessell, M. S., et al. 2013, ApJ, 762, 26

Zacharias, N., Finch, C. T., Girard, T. M., et al. 2013, AJ, 145,44

Zacharias, N., Finch, C., \& Frouard, J. 2017, AJ, 153, 166 


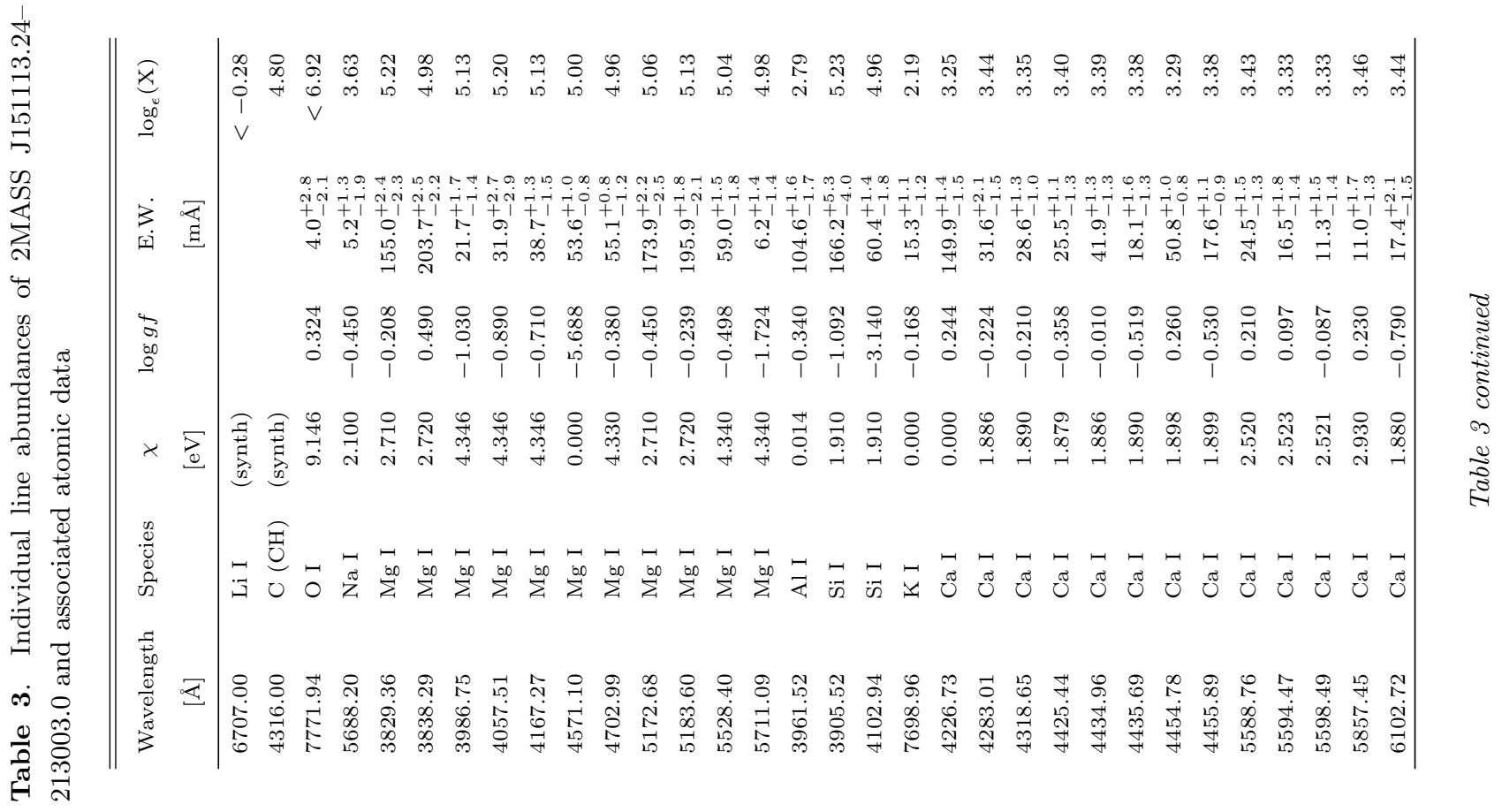




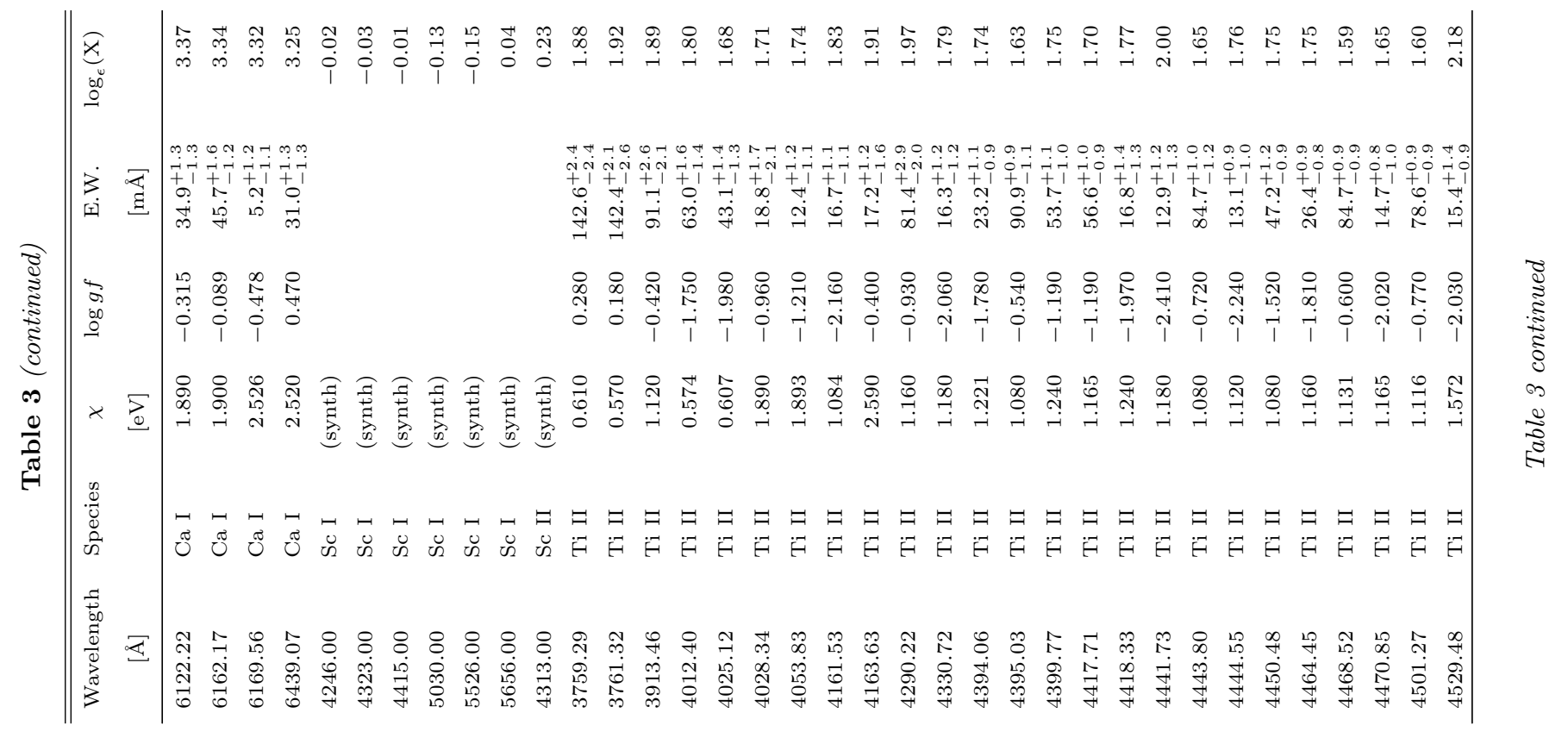




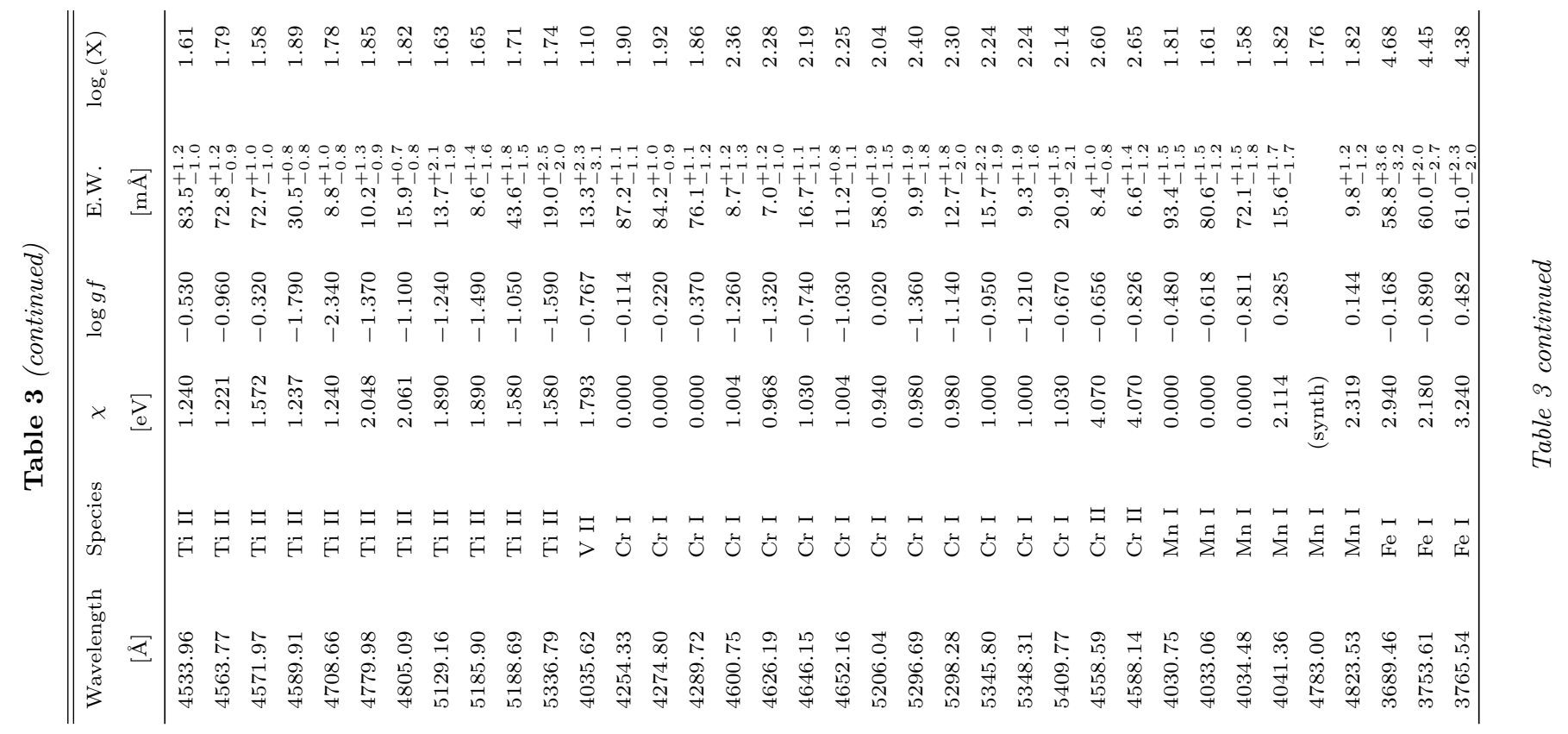




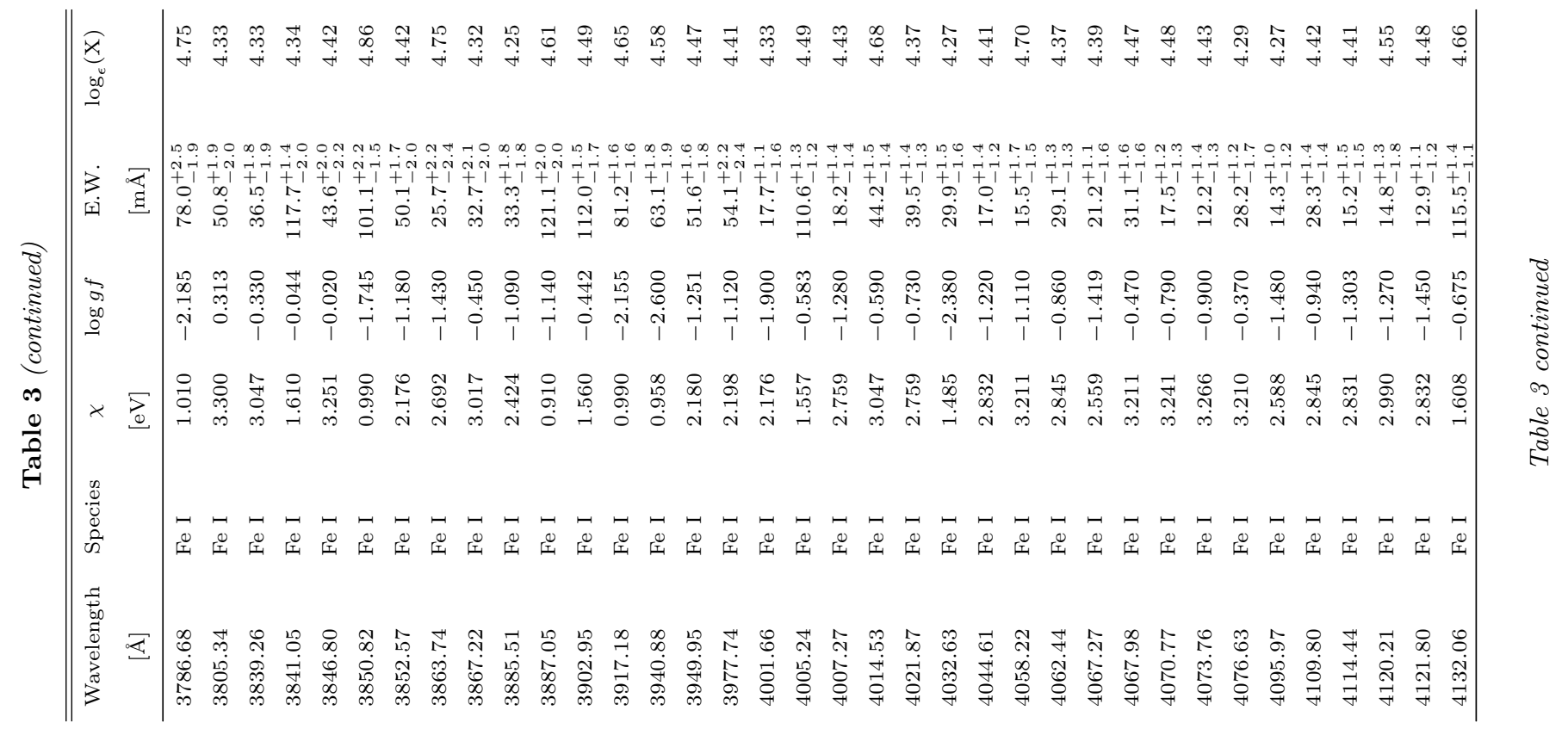




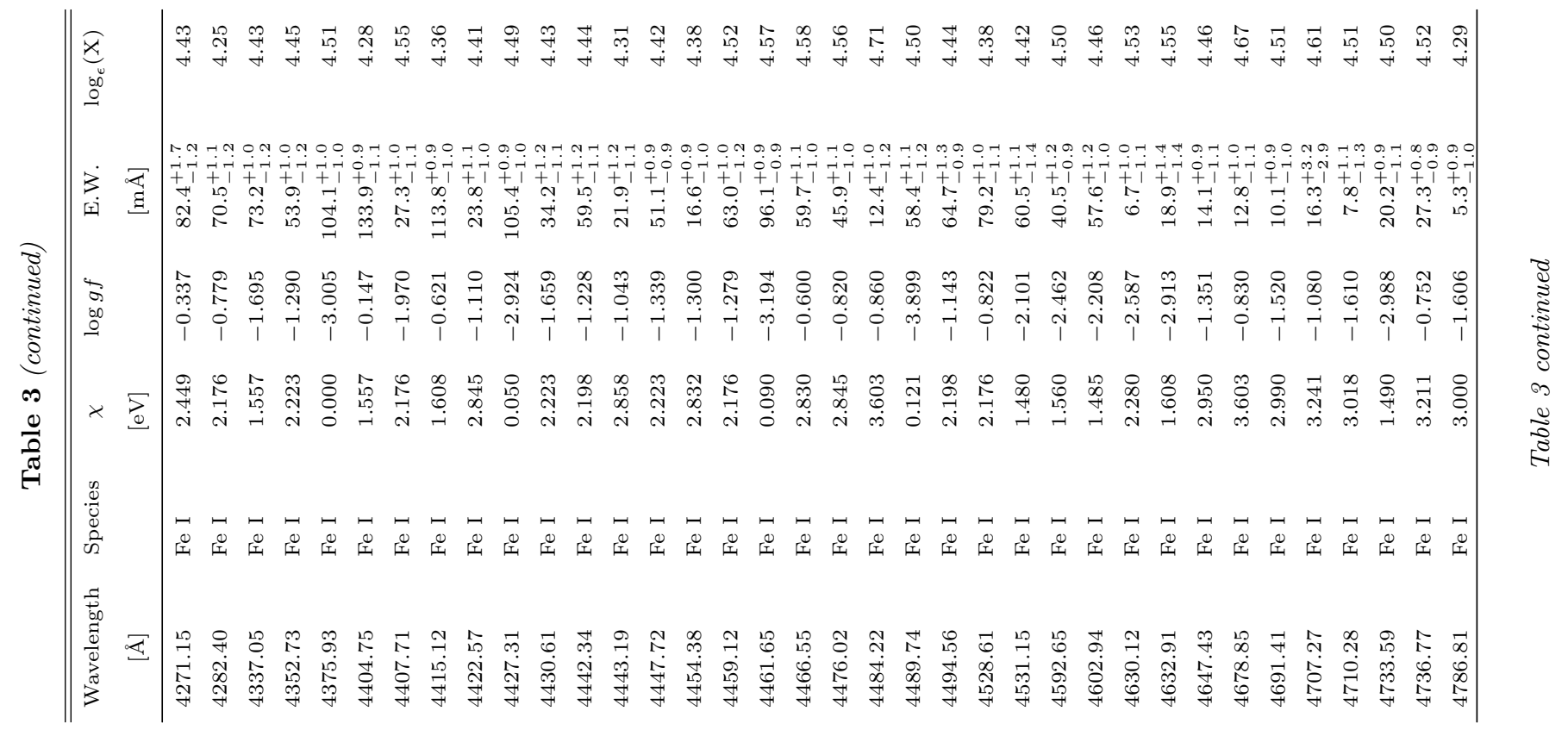




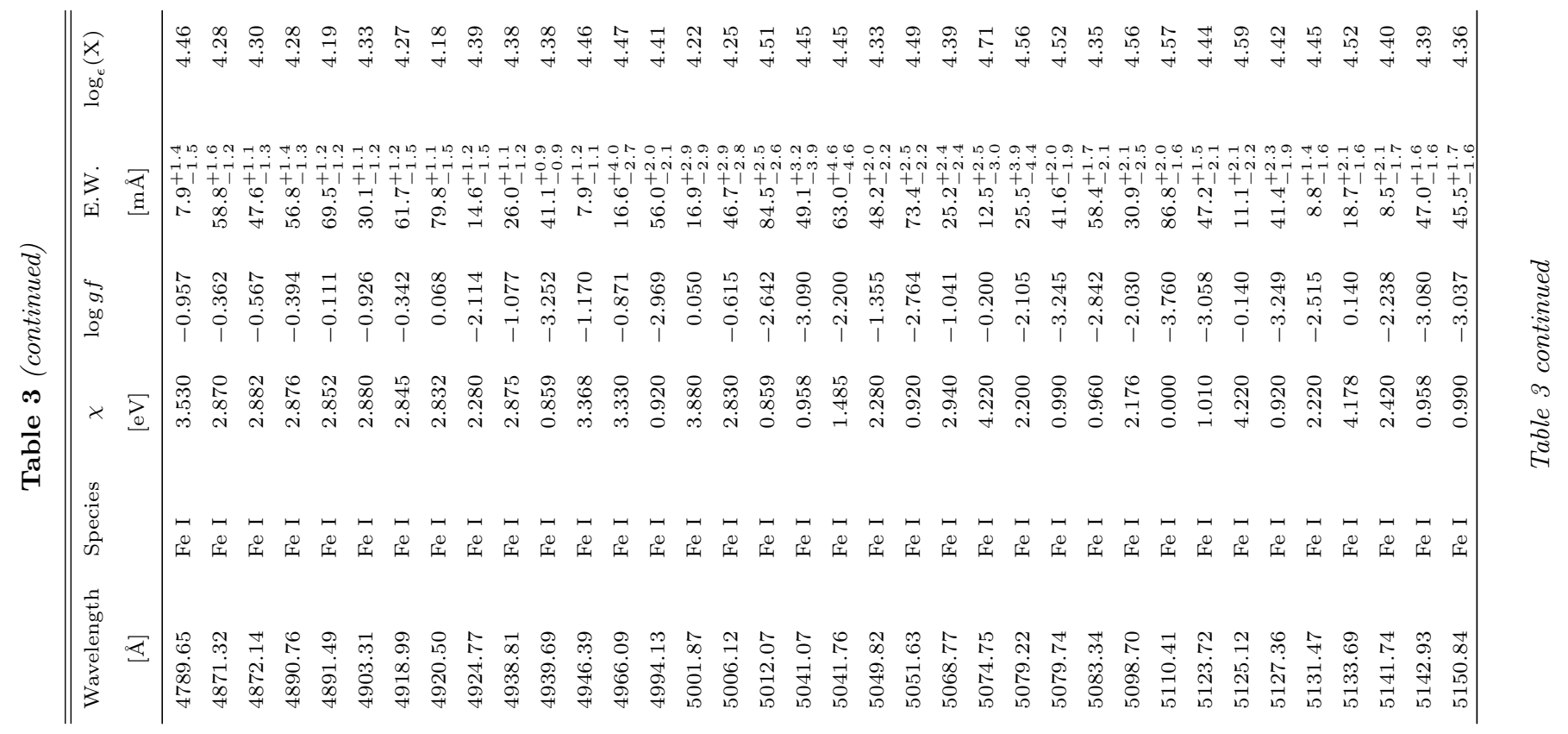




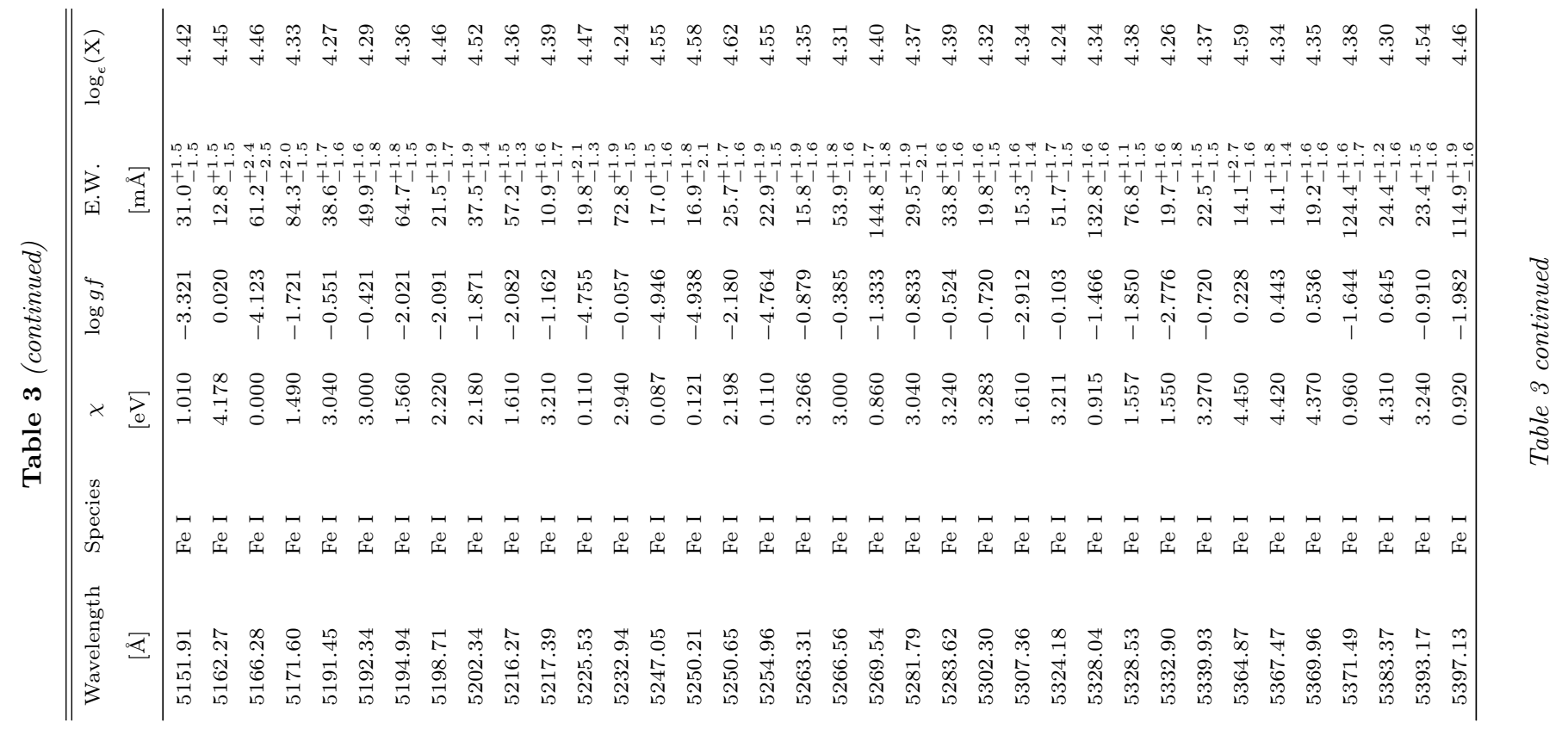




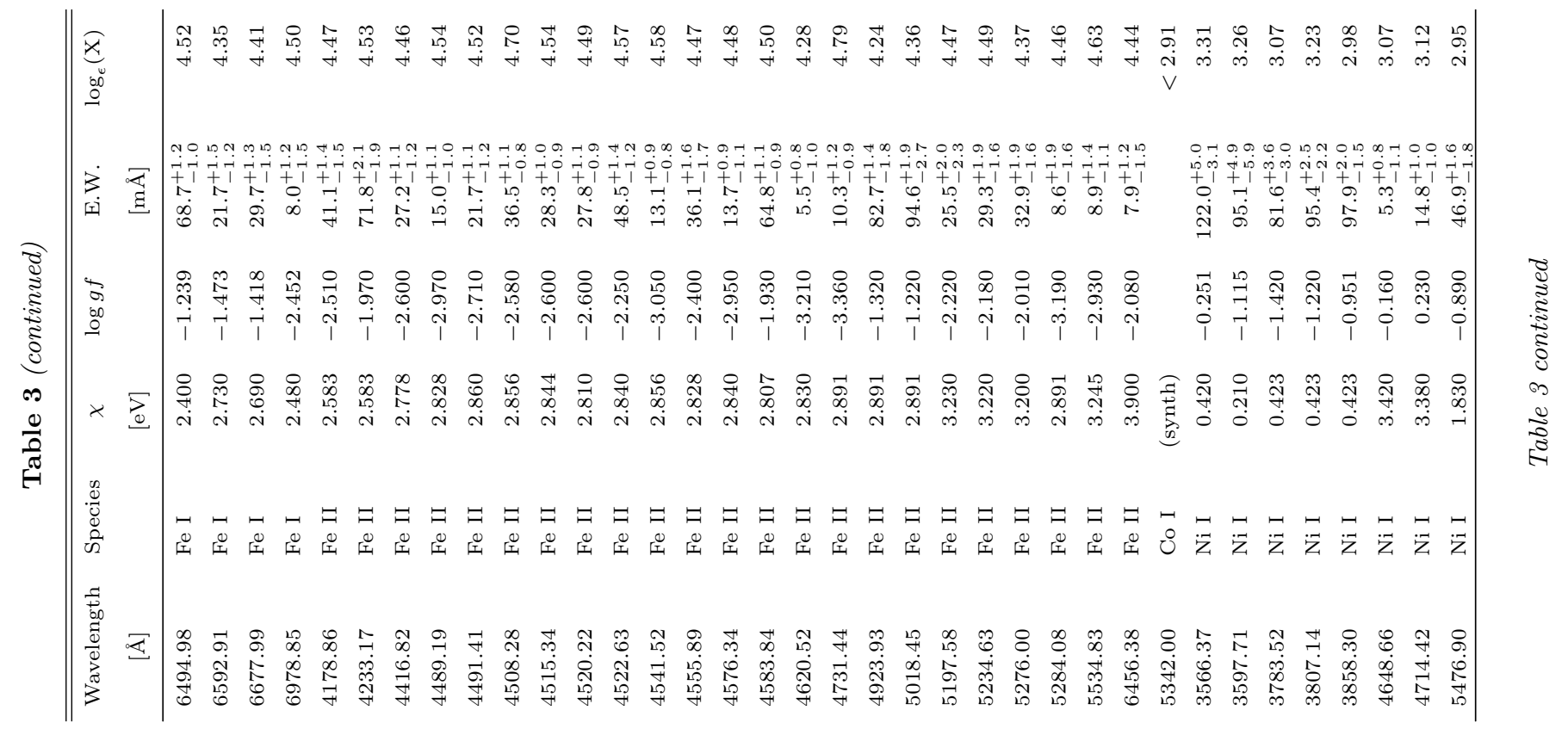




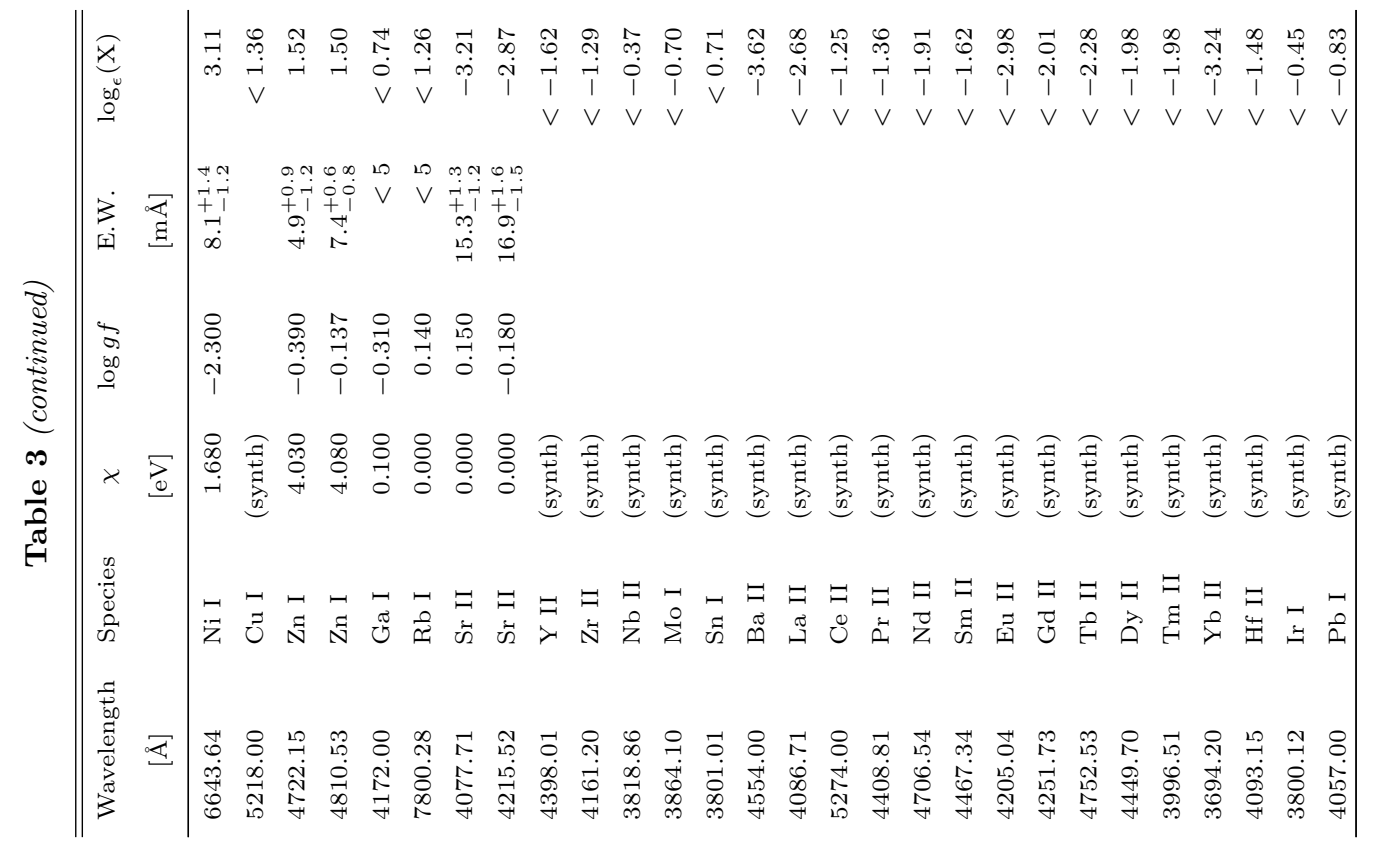

